\title{
Palinologia (esporos de fungos e pteridófitas, grãos de pólen de gimnoper- mas, cistos de algas e escolecodonte) das formações Solimões e Içá (Neoge- no e Pleistoceno, Bacia do Solimões), Amazonas, Brasil
}

\author{
Rosemery Rocha da SILVEIRA ${ }^{1,2} \&$ Paulo A. SOUZA ${ }^{2}$ \\ 1 Departamento de Geociências, Instituto de Ciências Exatas, Universidade Federal do Amazonas. Av. Gal. Rodrigo Jordão Ra- \\ mos, 3000, CEP 69077-000, Manaus, AM, Brasil. E-mail: rrsilveira@ufam.edu.br. \\ 2 Programa de Pós-Graduação em Geociências, Laboratório de Palinologia Marleni Marques Toigo, Instituto de Geociências, \\ Universidade Federal do Rio Grande do Sul. Av. Bento Gonçalves, 9500, CEP 91540-000, Porto Alegre, RS, Brasil. E-mail: paulo. \\ alves.souza@ufrgs.br.
}

Recebido em 10/2014. Aceito para publicação em 07/2015.

Versão online publicada em 23/02/2016 (www.pesquisasemgeociencias.ufrgs.br)

\begin{abstract}
Resumo- 0 conhecimento palinológico dos depósitos das formações cenozoicas da Bacia do Solimões tem sido substancialmente aprimorado nas últimas décadas, a partir de materiais de subsuperfície, bem como de afloramentos. Este trabalho apresenta parte dos resultados taxonômicos do estudo de 93 amostras oriundas de 12 afloramentos das formações Solimões e Içá, na região de Coari (cinco afloramentos), e Alto Solimões (sete afloramentos), estado do Amazonas, Brasil, que revelaram conjuntos palinológicos abundantes, diversificados e bem preservados. Aqui são listadas e ilustradas 53 espécies de palinomorfos, compreendendo 11 espécies de esporos de fungos, 31 de esporos de pteridófitas e dois de grãos de pólen de gimnospermas, oito de algas (sete clorofíceas e uma dinofícea), e um de escolecodonte, com descrições quando necessário. A composição palinológica é distinta para as duas áreas analisadas, tendo na região do Alto Solimões documentação de formas como Crassoretitriletes vanraadshooveni, Foveotriletes ornatos, Magnastriatites grandiosus e a ocorrência de um cisto de dinoflagelado. Na região de Coari foi verificado predomínio de esporomorfos, com grande diversidade e abundância de esporos de fungo e pteridófitas.

Palavras-chave: palinomorfos, Neogeno, Pleistoceno, Formação Solimões, Formação Içá, Amazonas.
\end{abstract}

\begin{abstract}
PALYNOLOGY (FUNGI AND FERN SPORES, GIMNOSPERM POLLEN GRAINS, ALGAE CYSTS AND SCOlecodont) From the Solimões and Içá formations (Neogene and Pleistocene, Solimões BASIN), AMAZONAS, BRAzIL. The palynological knowledge from Cenozoic units of the Solimões Basin has been substantially improved in last decades, based on subsurface and outcropping sampling. This work presents the taxonomic results from the study of 93 samples from 12 outcrops of Solimões and Içá formations, in the region of Coari (5 outcrops), and Alto Solimões (7 outcrops), state of Amazonas, Brazil, which revealed diversified, abundant and well preserved palynological assemblages. Here 53 species of palynomorphs recorded, comprising 11 fungi spores species, 31 fern spores, two pollen grains of gymnosperms, eight algae (seven chlorophyceae, one dinophyceae) and one scolecodont are listed and illustrated, as well as described when needed. The composition of both palynofloras is distinct: the Alto Solimões associations include Crassoretitriletes vanraadshooveni, Foveotriletes ornatus, Magnastriatites grandiosus and Multimarginites vanderhammenii, as well a dinoflagellate cyst. In the region of Coari it was recorded predominance of sporomorphs, with great diversity and abundance of fungi and ferns spores.
\end{abstract}

Keywords: palinomorphs, Neogene, Pleistocene, Solimões Formation, Içá Formation, Amazonas.

\section{Introdução}

Os estudos palinológicos dos depósitos neogenos a pré-holocenos da América do Sul foram iniciados a partir de meados do século XX (Germeraad et al., 1968; Regali, 1971; Regali et al., 1974ab; Cruz, 1984; Lorente, 1986; Muller et al., 1987), re- velando associações palinológicas diversificadas, cuja composição e padrões quantitativos têm sido significativos na resolução de questões bioestratigráficas, paleoambientais e paleoecológicas.

De forma geral, as contribuições palinológicas mais numerosas no Brasil para depósitos de idades cenozoicas estão relacionados ao Rift Continental 
do Sudeste Brasileiro (RCSB), nas bacias de Itaboraí (Lima \& Cunha, 1986), Macacu (Lima et al., 1996), Volta Redonda (Lima et al., 1994), Resende (Lima \& Amador, 1985; Lima \& Melo, 1994; Yamamoto, 1995), Taubaté ( Lima et al., 1985ab; Yamamoto, 1995; Mercês 2005; Mercês et al., 2006), Bonfim (Lima \& Dino, 1984), São Paulo (Lima et al., 1991; Yamamoto, 1995; Santos, 2005, Santos et al., 2006ab; Garcia et al., 2007; Santos et al., 2010), Tanque (Garcia et al., 2008), Curitiba (Garcia et al., 2013) e Formação Alexandra (Lima \& Angulo, 1990), além das bacias de Aiuruoca (Garcia et al, 2000; Ramos, 2013), Gandarela e Fonseca (Lima \& Salard-Cheboldaeff, 1981; Maizatto, 2001).

0 conhecimento sobre a paleoflora dessas idades do norte da América do Sul é advindo dos dados palinológicos, com geração de modelos paleovegetacionais para distintos intervalos de tempo (Germeraad et al., 1968; Cruz, 1984; Lorente, 1986; Muller et al., 1987; Hoorn, 1993, 1994ab; Carvalho, 1996; Leite et al., 1997ab; Leite, 2004, 2006; Silveira, 2005; Silva-Caminha et al., 2010; Nogueira et al., 2013).

Depósitos meso-neomiocenos na porção oeste do estado do Amazonas foram interpretados em um contexto paleoambiental continental, com influência marinha episódica (Hoorn, 1993; Leite, 2006). Contudo um trabalho recente realizado em área muito próxima destes mencionados contesta a influência marinha para os depósitos neomiocenos (Silva-Caminha et al., 2010). Associações neomiocenas-pliocenas (Cruz, 1984; Silveira, 2005; Silveira \& Nogueira, 2007; Silva-Caminha et al., 2010; Nogueira et al., 2013) e pleistocenas (Silveira, 2005; Nogueira et al., 2013) da Amazônia ocidental e central foram mais bem estudadas nos últimos anos, revelando palinomorfos exclusivamente continentais.

Assembleias bem preservadas e diversificadas foram recuperadas de amostras de afloramentos das formações Solimões e Içá, nas regiões de Coari e Alto Solimões. Neste trabalho são apresentados os esporos de fungos e de pteridófitas, grãos de pólen de gimnospermas, cistos de algas (clorofíceas e dinofícea) e um escolecodonte recuperados nestas seções, como contribuição para o entendimento da diversidade palinológica da região entre o Mesomioceno e o Pleistoceno.

\section{2 Área, materiais e métodos}

\subsection{Contexto geológico}

A Bacia do Solimões compreende uma área de aproximadamente $440.000 \mathrm{~km}^{2}$, totalmente inseri- da no estado do Amazonas, subdividida pelo Arco de Carauari em duas sub-bacias: Juruá, a leste, e Jandiatuba, a oeste. Apresenta limite ao norte com o Escudo das Guianas, a sul com o Escudo Brasileiro, a oeste o Arco de Iquitos a separa da Bacia do Acre e demais bacias andinas; a leste é separada da Bacia do Amazonas pelo Arco de Purus (Caputo, 1984; Eiras et. al., 1994; Wanderley Filho et al., 2007).

A última sequência deposicional da bacia corresponde ao Grupo Javari, que compreende as formações Alter do Chão (Cretáceo Superior) e Solimões (Neogeno), separadas por discordância erosiva. Terraços fluviais quaternários mais jovens recobrem localmente esta última unidade (Caputo, 1984; Eiras et. al., 1994; Wanderley Filho et al., 2007). Contudo, ainda que não sejam reconhecidas nas cartas estratigráficas das bacias amazônicas vigente, há ocorrência de arenitos amarelo-avermelhados, finos a conglomeráticos da Formação Içá, e que na Sub-bacia do Juruá, ocorre entre os depósitos da Formação Solimões e os estratos quaternários, e que é relacionado a um sistema fluvial meandrante.

Daemon \& Contreiras (1971) posicionaram a Formação Solimões entre o Paleoceno e o Pleistoceno. Cruz (1984) admitiu, para a unidade na porção central da Amazônia, uma idade entre Eomioceno ao Plioceno. As análises palinológicas de Hoorn (1993) revelaram conjuntos palinológicos do Eomioceno ao Meso-Neomioceno. Trabalhos mais recentes, ambos desenvolvidos na porção ocidental e oriental da Amazônia brasileira (Silveira, 2005; Leite, 2006; Silveira \& Nogueira, 2007; Silva-Caminha et al., 2010), apontam idades compatíveis àquela estabelecida por Cruz (1984).

Melo \& Villas Boas (1993) adotaram a denominação de Maia et al. (1977), para a Formação Içá, para depósitos que correspondem à cobertura quaternária, constituídos de arenitos amarelo-avermelhados, finos a conglomeráticos, friáveis, tipicamente continentais, que se estendem por grande parte da área do Alto Rio Negro, e que na região de Coari tem sua idade representada por associação palinológicas do Pleistoceno (Silveira, 2005; Nogueira et al., 2013; Silveira, 2015; Silveira \& Souza, 2015). Essa mesma unidade é reportada na sub-bacia do Jandiatuba (Maia et al., 1977) e na Bacia do Amazonas (Soares et al. 2010).

\subsection{Amostragem}

A amostragem é advinda de cinco afloramentos designados P2, P9, P11, P12 e P13, localizados às margens do rio Solimões (Fig. 1B), descritos por 


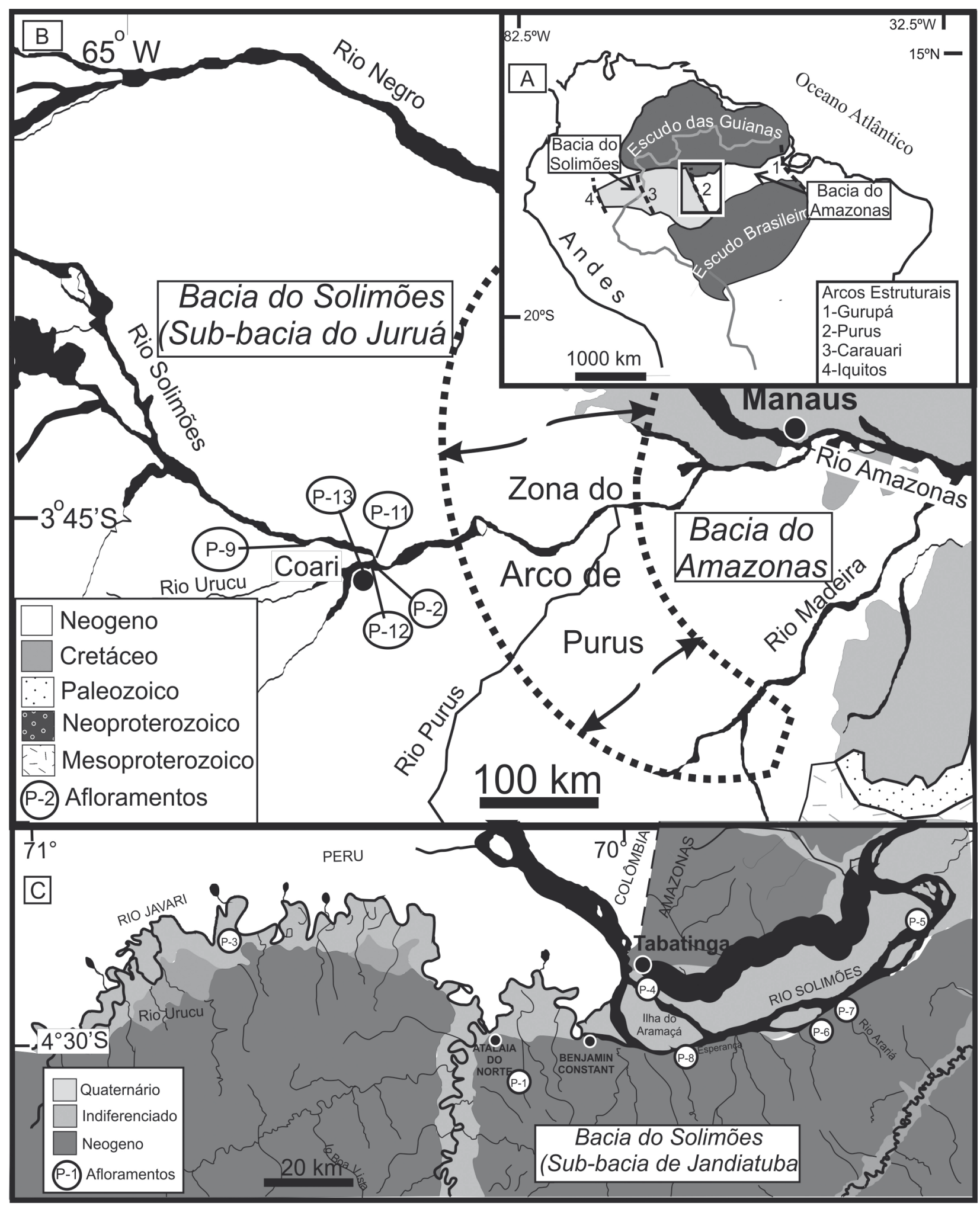

Figura 1. Localização da área de amostragem: A) Mapa geológico; B) Localização da área estudada e amostragem na região de Coari (pontilhado em B corresponde a limite impreciso do Arco de Purus, Sub-bacia do Juruá (modificado de Nogueira et al., 2013);, C) Localização pontos de coleta do Alto Solimões na Sub-bacia do Jandiatuba, Bacia do Solimões.

Nogueira et al. (2013), de áreas próximas ao município de Coari, bem como sete afloramentos, nomeados de P1, P3, P4, P5, P6, P7 e P8, reportados por Silveira (2015), às margens dos rios Javari e Solimões, aqui chamada de região do Alto Solimões, para as localidades adjacentes aos municípios de
Atalaia do Norte e Tabatinga, no estado do Amazonas (Fig. 1C). As amostras foram coletadas durante o período de vazante, entre setembro e outubro de 2003 (levantamento de Coari) e entre outubro e novembro de 2009 (região do Alto Solimões).

As 93 amostras coletadas nas duas regiões 
foram encaminhadas para o Laboratório de Paleontologia da Universidade Federal do Amazonas para secagem, pesagem e trituração. Após tratamento mecânico, 52 amostras foram destinadas ao Centro de Pesquisa e Desenvolvimento Leopoldo Américo Miguez de Mello (CENPES/PETROBRÁS), onde seguiram o processamento palinológico de acordo com Uesugui (1979). As demais 41 amostras foram tratadas no Laboratório de Palinologia Marleni Marques Toigo do Instituto de Geociências da Universidade Federal do Rio Grande do Sul (LPMMT/UFRGS), submetidas a etapas de processamento ligeiramente distintas, como sintetizado por Silva et al. (2011). Em ambos os processamentos foram empregados peneiramento com malhas de $10 \mu \mathrm{m}$.

O concentrado obtido das amostras de Coari foi utilizado para a confecção de 156 lâminas palinológicas (três lâminas por nível estratigráfico) analisadas quantitativa e qualitativamente. As lâminas foram arquivadas no Laboratório de Paleontologia do Departamento de Geociências da Universidade Federal do Amazonas. Os concentrados obtidos das amostras do Alto Solimões foram utilizados para a confecção de 129 lâminas palinológicas (variando de dois a quatro lâminas por nível estratigráfico), depositadas no LPMMT/UFRGS.

\section{Resultados}

Das 93 amostras processadas, 82 mostraram resultados palinológicos satisfatórios, com diferentes concentrações e grau de preservação de palinomorfos. Em todos os níveis amostrados para a região de Coari foram observados espécimes com baixa preservação, em particular os esporos de parede psilada, e melhor preservação nos esporos de parede ornamentada. Os palinomorfos provenientes da região do Alto Solimões apresentam boa preservação, raramente ocorrendo rasgados.

Um total de 101 táxons de palinomorfos foi reconhecido, incluindo 11 esporos de fungos, oito de algas (sete clorofíceas e uma dinofícea), um escolecodonte, , 31 esporos de pteridófitas e 48 grãos de pólen de angiospermas e dois de giminospermas. Neste trabalho são apresentadas medidas, ocorrência, afinidade botânica e outras informações ecológicas para todos esses palinomorfos, excetuando-se aqueles relativos aos grãos de pólen de angiospermas, registrados separadamente em Silveira \& Souza (2015). Descrições são exclusivas para aqueles com taxonomia aberta.

Para a sistemática dos grãos de pólen e esporos foram adotados os trabalhos de classificação de H. Potonié (1893) e R. Potonié (1956; 1960).
Fotomicrografias de espécimes selecionados são apresentadas nas figuras 2 a 6 . Informações sobre procedência e disposição nas lâminas palinológicas constam no apêndice.

\subsection{Esporos de fungos}

\section{Divisão EUMYCOTA \\ Classe FUNGI IMPERFECTI \\ Grupo AMEROSPORAE}

Monoporisporites (van der Hammen) Elsik 1978

\section{Monoporisporites sp. 1}

Fig. 2.1

Descrição: esporo unicelado, asseptado, colonial, monoporado, contorno circular, psilado; poro com ânulo com $3 \mu \mathrm{m}$ de diâmetro, espessura do ânulo 3-3,5 $\mu \mathrm{m}$.

Dimensões (512 espécimes): menor comprimento 23 a $30 \mu \mathrm{m}$, maior comprimento 24 a $32 \mu \mathrm{m}$.

Afinidade biológica: Xylariaceae (Guimarães et al., 2013).

Ocorrência: formações Solimões (Coari: P2, P9, P11, P12, P13) e Içá (Coari, P2).

\section{Monoporisporites sp. 2}

Fig. 2.2

Descrição: esporo unicelado, asseptado, monoporado, contorno elíptico, psilado; poro 3 $\mu \mathrm{m}$ de diâmetro.

Dimensões (793 espécimes): menor comprimento 18 a $23 \mu \mathrm{m}$, maior comprimento 25 a $32 \mu \mathrm{m}$.

Afinidade biológica: Xylariaceae.

Observação: diferencia-se de Monoporisporites sp. 1 pelo contorno elíptico.

Ocorrência: formações Solimões (Coari: P2, P13) e Içá (Coari, P2).

\section{Monoporisporites sp. 3}

Fig. 2.3

Descrição: esporo unicelado, asseptado, monoporado, com contorno elíptico, psilado; poro com 5 um de diâmetro.

Dimensões (190 espécimes): menor comprimento 47 a $50 \mu \mathrm{m}$, maior comprimento 70 a $75 \mu \mathrm{m}$.

Afinidade biológica: Xylariaceae.

Ocorrência: formações Solimões (Coari: P2, P9, P13) e Içá (Coari: P2)

\section{Foveodiporites Varma \& Rawat 1963}


Quadro 1. Distribuição estratigráfica dos palinomorfos estudados nos afloramentos de Coari e Alto Solimões.

\begin{tabular}{|c|c|c|c|c|c|c|c|c|c|c|c|c|c|}
\hline \multirow{3}{*}{ Táxon } & \multicolumn{6}{|c|}{ Coari } & \multicolumn{7}{|c|}{ Alto Solimões } \\
\hline & \multirow{2}{*}{$\frac{\text { Fm. Içá }}{\text { P2 }}$} & \multicolumn{5}{|c|}{ Fm. Solimões } & \multicolumn{7}{|c|}{ Fm. Solimões } \\
\hline & & $\mathrm{P} 2$ & P9 & P11 & P12 & $\mathrm{P} 13$ & P1 & $\mathrm{P} 3$ & $\mathrm{P} 4$ & $\mathrm{P} 5$ & P6 & P7 & P8 \\
\hline \multicolumn{14}{|l|}{ Esporos de fungos } \\
\hline Diporicellaesporites sp. 1 & $\mathrm{X}$ & & & & & & & & & & & & \\
\hline Diporicellaesporites sp. 2 & $\mathrm{X}$ & & & & & & & & & & & & \\
\hline $\begin{array}{l}\text { Esporo de fungo } \\
\text { indeterminado }\end{array}$ & $\mathrm{X}$ & $\mathrm{X}$ & & & & & & & & & & & \\
\hline Foveodiporites sp. & & $\mathrm{X}$ & & & & & & & & & & & \\
\hline Fusiformisporites crabbii & $\mathrm{X}$ & $\mathrm{X}$ & $\mathrm{X}$ & & & $\mathrm{X}$ & & & & & & & \\
\hline Mediaverrunites elsekii & $\mathrm{X}$ & $\mathrm{X}$ & & & & & & & & & & & \\
\hline Meliolinites sp. & $\mathrm{X}$ & $\mathrm{X}$ & $X$ & & & & & & & & & & \\
\hline Monoporisporites sp. 1 & $\mathrm{X}$ & $\mathrm{X}$ & $\mathrm{X}$ & $\mathrm{X}$ & $\mathrm{X}$ & $\mathrm{X}$ & & & & & & & \\
\hline Monoporisporites sp. 2 & $\mathrm{X}$ & $\mathrm{X}$ & & & & $\mathrm{X}$ & & & & & & & \\
\hline Monoporisporites sp. 3 & $\mathrm{X}$ & $\mathrm{X}$ & $\mathrm{X}$ & & & $\mathrm{X}$ & & & & & & & \\
\hline Quilonia sp. & $\mathrm{X}$ & $\mathrm{X}$ & $\mathrm{X}$ & & $\mathrm{X}$ & $\mathrm{X}$ & & & & & & & \\
\hline \multicolumn{14}{|l|}{ Esporos de pteridófitas } \\
\hline Cicatricosisporites sp. & $\mathrm{X}$ & $\mathrm{X}$ & & & & & & & & & & & \\
\hline Cingulatisporites sp. & & $\mathrm{X}$ & $X$ & & $\mathrm{X}$ & $\mathrm{X}$ & $\mathrm{X}$ & $\mathrm{X}$ & & & $\mathrm{X}$ & & \\
\hline $\begin{array}{l}\text { Crassoretitriletes } \\
\text { vanraadshoovenii }\end{array}$ & & & & & & & $X$ & $\mathrm{X}$ & $\mathrm{X}$ & $\mathrm{X}$ & $X$ & $\mathrm{X}$ & $\mathrm{X}$ \\
\hline Cyatheacidites annulatus & $\mathrm{X}$ & $\mathrm{X}$ & & & & & & & & & & & \\
\hline Cyathidites sp. 1 & $\mathrm{X}$ & $\mathrm{X}$ & $\mathrm{X}$ & $\mathrm{X}$ & $\mathrm{X}$ & $\mathrm{X}$ & & & & & & & \\
\hline Cyathidites sp. 2 & $\mathrm{X}$ & $\mathrm{X}$ & $\mathrm{X}$ & & $\mathrm{X}$ & $\mathrm{X}$ & & & & & & & \\
\hline Cyathidites sp. 3 & & & & & & & & & & & $\mathrm{X}$ & & \\
\hline Deltoidospora adriennis & $\mathrm{X}$ & $\mathrm{X}$ & $\mathrm{X}$ & & $\mathrm{X}$ & $\mathrm{X}$ & $\mathrm{X}$ & $\mathrm{X}$ & $\mathrm{X}$ & $\mathrm{X}$ & $\mathrm{X}$ & $\mathrm{X}$ & $\mathrm{X}$ \\
\hline Echitriletes muelleri & $\mathrm{X}$ & $\mathrm{X}$ & $\mathrm{X}$ & $\mathrm{X}$ & $\mathrm{X}$ & $\mathrm{X}$ & $\mathrm{X}$ & & & & & $\mathrm{X}$ & \\
\hline Foveotriletes ornatus & & & & & & & $\mathrm{X}$ & & & & & & \\
\hline Kuylisporites waterbolkii & & $\mathrm{X}$ & $\mathrm{X}$ & $\mathrm{X}$ & $\mathrm{X}$ & $\mathrm{X}$ & $\mathrm{X}$ & & & & & & \\
\hline Laevigatosporites sp. 1 & $\mathrm{X}$ & $\mathrm{X}$ & & & & & & & & & & & \\
\hline Laevigatosporites sp. 2 & $\mathrm{X}$ & $\mathrm{X}$ & $\mathrm{X}$ & $\mathrm{X}$ & $\mathrm{X}$ & $\mathrm{X}$ & & $\mathrm{X}$ & & & & & \\
\hline Laevigatosporites sp. 3 & $\mathrm{X}$ & $\mathrm{X}$ & $\mathrm{X}$ & $\mathrm{X}$ & $\mathrm{X}$ & $\mathrm{X}$ & & & & & & & \\
\hline Laevigatosporites sp. 4 & $\mathrm{X}$ & $\mathrm{X}$ & $\mathrm{X}$ & $\mathrm{X}$ & $\mathrm{X}$ & $\mathrm{X}$ & & & & & & & \\
\hline Laevigatosporites vulgaris & $\mathrm{X}$ & $\mathrm{X}$ & $\mathrm{X}$ & & $\mathrm{X}$ & $\mathrm{X}$ & & & & & & & \\
\hline Magnastriatites grandiosus & $\mathrm{X}$ & $\mathrm{X}$ & $\mathrm{X}$ & & $\mathrm{X}$ & $\mathrm{X}$ & $\mathrm{X}$ & $\mathrm{X}$ & $\mathrm{X}$ & $\mathrm{X}$ & $\mathrm{X}$ & $\mathrm{X}$ & $\mathrm{X}$ \\
\hline Perinomonoletes sp. & $\mathrm{X}$ & $\mathrm{X}$ & $\mathrm{X}$ & $\mathrm{X}$ & $\mathrm{X}$ & $\mathrm{X}$ & & & & & & & \\
\hline Polypodiaceiosporites potoniei & $\mathrm{X}$ & $\mathrm{X}$ & $\mathrm{X}$ & $\mathrm{X}$ & $\mathrm{X}$ & $\mathrm{X}$ & & $\mathrm{X}$ & $\mathrm{X}$ & & & $\mathrm{X}$ & $\mathrm{X}$ \\
\hline P. pseudopsilatus & $\mathrm{X}$ & $\mathrm{X}$ & $\mathrm{X}$ & & & & & & & & & & \\
\hline Polypodiaceiosporites sp. & & $\mathrm{X}$ & & & & & & & & & & & \\
\hline Polypodiisporites radiatus & & $\mathrm{X}$ & $\mathrm{X}$ & & $\mathrm{X}$ & & & & & & & & \\
\hline Psilatriletes peruanus & & $\mathrm{X}$ & $\mathrm{X}$ & & $\mathrm{X}$ & $\mathrm{X}$ & & $\mathrm{X}$ & $\mathrm{X}$ & $\mathrm{X}$ & & $\mathrm{X}$ & $\mathrm{X}$ \\
\hline Striatriletes saccolomoides & & & & & & $\mathrm{X}$ & $\mathrm{X}$ & & & & & & \\
\hline Verrucatosporites favus & $\mathrm{X}$ & $\mathrm{X}$ & $\mathrm{X}$ & $\mathrm{X}$ & $\mathrm{X}$ & $\mathrm{X}$ & & & & & & & \\
\hline Verrucatosporites sp. 1 & $\mathrm{X}$ & $\mathrm{X}$ & $\mathrm{X}$ & & $\mathrm{X}$ & & & & & & & & \\
\hline Verrucatosporites sp. 2 & $\mathrm{X}$ & $\mathrm{X}$ & $\mathrm{X}$ & & $\mathrm{X}$ & & & & & & & & \\
\hline Verrucatosporites usmensis & & & & $\mathrm{X}$ & & $\mathrm{X}$ & $\mathrm{X}$ & $\mathrm{X}$ & $\mathrm{X}$ & & $\mathrm{X}$ & $\mathrm{X}$ & $\mathrm{X}$ \\
\hline Verrucatotriletes bullatus & $\mathrm{X}$ & $\mathrm{X}$ & $\mathrm{X}$ & & $\mathrm{X}$ & & & & & & & & \\
\hline Verrucatotriletes sp. 1 & $\mathrm{X}$ & $\mathrm{X}$ & $\mathrm{X}$ & & $\mathrm{X}$ & $\mathrm{X}$ & & & & & & & \\
\hline Verrucatotriletes sp. 2 & $\mathrm{X}$ & $\mathrm{X}$ & $\mathrm{X}$ & & $\mathrm{X}$ & $\mathrm{X}$ & & & & & & & \\
\hline \multicolumn{14}{|l|}{ Grão de pólen de gimnospermas } \\
\hline Podocarpidites sp. 1 & & & $\mathrm{X}$ & $\mathrm{X}$ & $\mathrm{X}$ & $\mathrm{X}$ & $\mathrm{X}$ & & & & & & \\
\hline Podocarpidites sp. 2 & & & & & & & $\mathrm{X}$ & $\mathrm{X}$ & & & & $\mathrm{X}$ & \\
\hline \multicolumn{14}{|l|}{ Algas clorofíceas } \\
\hline Ovoidites sp. & & $\mathrm{X}$ & $\mathrm{X}$ & $\mathrm{X}$ & $\mathrm{X}$ & $\mathrm{X}$ & & & & & & & \\
\hline Pediastrum sp. 1 & & & & & & & & $\mathrm{X}$ & & & & $\mathrm{X}$ & \\
\hline Pediastrum sp. 2 & & $\mathrm{X}$ & $\mathrm{X}$ & & $\mathrm{X}$ & $\mathrm{X}$ & & $\mathrm{X}$ & & & & $\mathrm{X}$ & \\
\hline Pediastrum sp. 3 & & & & & & & & $\mathrm{X}$ & & & & $\mathrm{X}$ & \\
\hline Pediastrum sp. 4 & & & & & & & & $\mathrm{X}$ & & & & $\mathrm{X}$ & \\
\hline Pseudoschizaea circulus & & $\mathrm{X}$ & $\mathrm{X}$ & & $\mathrm{X}$ & $\mathrm{X}$ & & & & & & & \\
\hline Pseudoschizaea sp. & & $\mathrm{X}$ & $\mathrm{X}$ & & $\mathrm{X}$ & $\mathrm{X}$ & & & & & & & \\
\hline \multicolumn{14}{|l|}{ Alga dinofícea } \\
\hline Diphyes sp. & & & & & & & & & & & & $\mathrm{X}$ & \\
\hline Fragmento de invertebrado & & & & & & & & & & & & & \\
\hline Escolecodonte indeterminado & & $\mathrm{X}$ & & & & & & & & & & & \\
\hline
\end{tabular}


Foveodiporites sp.

Fig. 2.4

Descrição: esporo unicelado, asseptado, diporado, com contorno elíptico; parede espessa e ornamentada por granulação incipiente por todo o esporo; presença de poros nas extremidades opostas.

Dimensões (6 espécimes): menor comprimento 20 a $23 \mu \mathrm{m}$, maior comprimento 47 a $52 \mu \mathrm{m}$.

Afinidade biológica: desconhecida.

Ocorrência: Formação Solimões (Coari: P2).

Fusiformisporites Rouse 1962

Fusiformisporites crabbii Rouse 1962

Fig. 2.5

Dimensões (137 espécimes): menor comprimento $20 \mu \mathrm{m}$, maior comprimento $40 \mu \mathrm{m}$.

Afinidade biológica: Cookeina, Sarcoscyphaceae (Kalgutkar \& Sigler, 1995; Kalgutkar \& Jansonius, 2000).

Ocorrência: formações Solimões (Coari: P2, P9, P13) e Içá (Coari: P2).

\section{Mediaverrusporonites Jarzen \& Elsik 1986}

Mediaverrusporonites elsikii Nandi \& Sinha 2007

Fig. 2.7

Dimensões (109 espécimes): menor comprimento $22 \mu \mathrm{m}$; maior comprimento $50 \mu \mathrm{m}$.

Afinidade biológica: desconhecida.

Ocorrência: formações Solimões (Coari: P2) e Içá (Coari: P2).

\section{Meliolinites Selkirk 1975}

\section{Meliolinites sp.}

Fig. 2.10

Descrição: esporo multicelado, cinco células de tamanhos irregulares, com 4 septos, contorno elíptico, com duas extremidades arredondadas, ornamentação psilada.

Dimensões (183 espécimes): menor comprimento $23 \mu \mathrm{m}$, maior comprimento $62 \mu \mathrm{m}$.

Afinidade biológica: desconhecida.

Ocorrência: formações Solimões (Coari: P2, P9) e Içá (Coari: P2).

Quilonia Jain \& Gupta 1970
Quilonia sp.

Fig. 2.11

Descrição: esporo multicelado, sete células de tamanhos irregulares, com 6 septos, contorno elíptico, com uma extremidade arredondada e outra afilada, ornamentação psilada.

Dimensões (127 espécimes): maior comprimento $52 \mu \mathrm{m}$, menor comprimento $20 \mu \mathrm{m}$.

Afinidade biológica: relaciona-se aos gêneros Corynespora, Sporidesmium, Cladosporium, Clasterosporim (Silveira, 1981).

Ocorrência: formações Solimões (Coari: P2, P9, P12, P13) e Içá (Coari, P2).

\section{Diporicellaesporites Elsik 1968}

\section{Diporicellaesporites sp. 1}

Fig. 2.8

Descrição: esporo de fungo tetracelado, com 3 septos, contorno elíptico, psilado; poros em cada extremidade.

Dimensões (3 espécimes): menor comprimento 16 a $18 \mu \mathrm{m}$, maior comprimento 26 a $28 \mu \mathrm{m}$.

Afinidade biológica: desconhecida.

Ocorrência: Formação Içá (Coari: P2).

\section{Diporicellaesporites sp. 2}

Fig. 2.9

Descrição: esporo de fungo tetracelado, com 3 septos, contorno elíptico, superfície granulada, terminações mais claras e alongadas; poros em cada extremidade.

Dimensões (1 espécime): menor comprimento 25 $\mu \mathrm{m}$, maior comprimento $63 \mu \mathrm{m}$.

Afinidade biológica: desconhecida.

Ocorrência: Formação Içá (Coari: P2).

\section{Esporo de fungo indeterminado}

Fig. 2.6

Descrição: esporo de fungo tricelado, com 2 septos, contorno elíptico, psilado; um poro em cada extremidade do esporo, com célula central mais escura.

Dimensões (12 espécimes): menor comprimento 19 a $22 \mu \mathrm{m}$, maior comprimento 27 a $32 \mu \mathrm{m}$.

Afinidade biológica: desconhecida.

Ocorrência: formações Solimões (Coari: P2) e Içá (Coari: P2). 

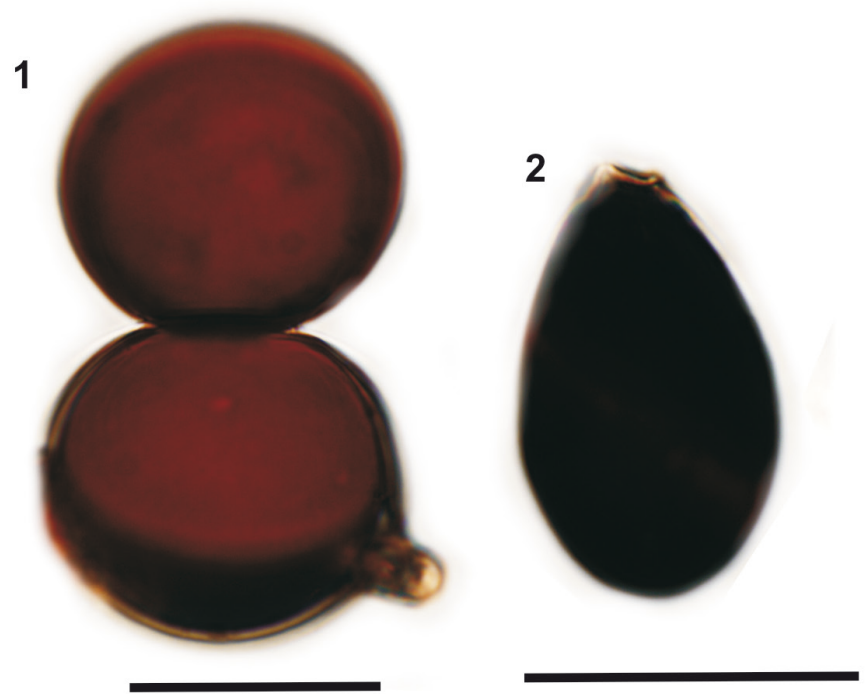

3
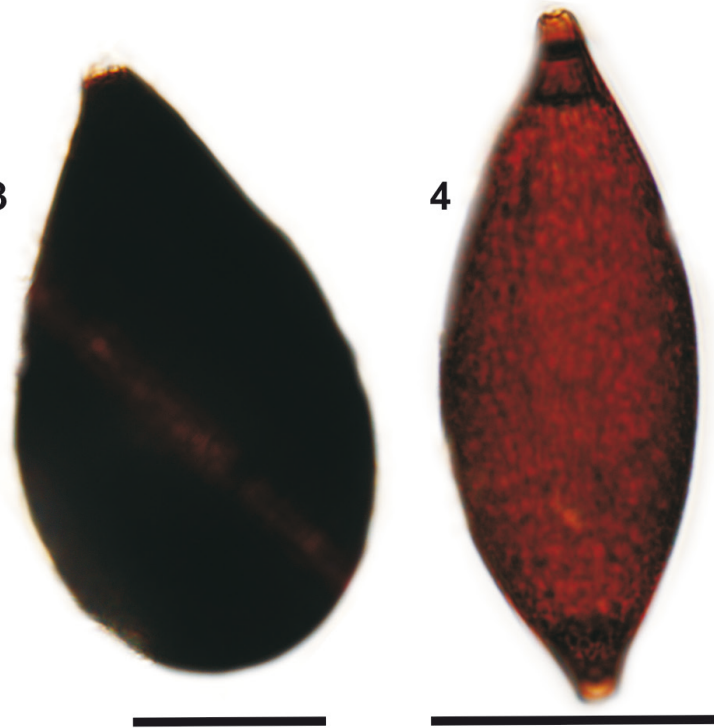

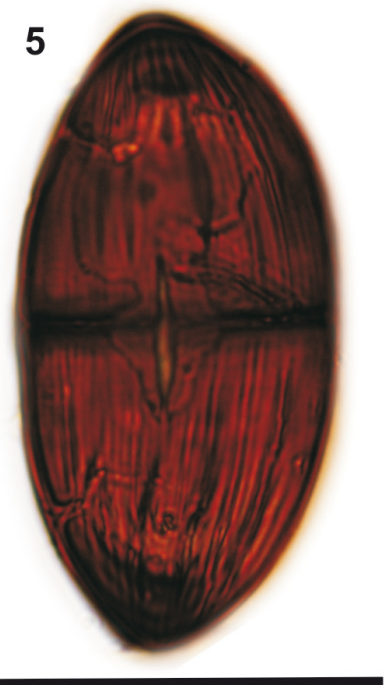

6

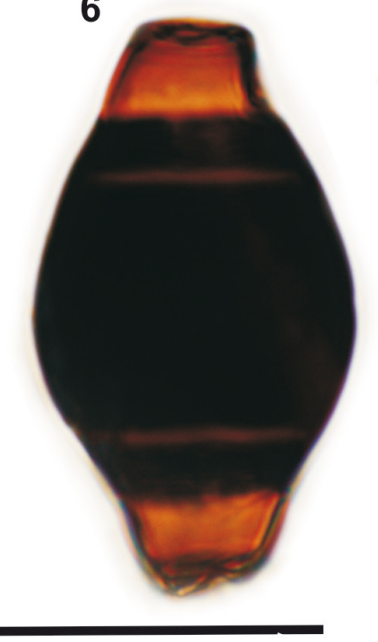

8

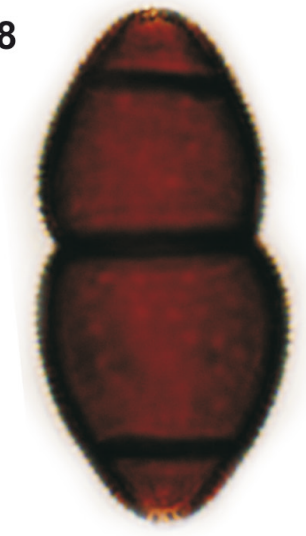

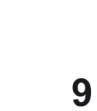

\section{9}

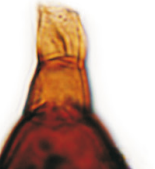




\subsection{Esporos de pteridófitas}

Divisão SPORITES Potonié 1893

Classe TRILETES Reinsch 1881 emend. Potonié \& Kremp 1954

Cyathidites Couper 1953

Cyathidites sp. 1

Fig. 3.3

Descrição: esporo simples, de tamanho médio, trilete, com exina psilada; radiossimétrico, âmbito triangular de lados côncavos e vértices arredondados, laesura com aproximadamente $3 / 4$ do raio do esporo.

Dimensões (322 espécimes): diâmetro equatorial 28(36) $44 \mu \mathrm{m}$, exina 0,5-0,7 $\mu \mathrm{m}$ de espessura.

Observações: a forma é similar a Concavissimisporites sp. de Singh (1964), todavia não apresenta superfície ondulada.

Afinidade botânica: Cyatheaceae, Cyathea (Tryon \& Tryon, 1982).

Informações ecológicas: as Cyatheaceae atuais são comuns em áreas montanhosas; a família ocorre como samambaias terrestres, muitas vezes de hábito arbóreo e, menos comumente, como epífitas ou trepadeiras (Tryon \& Tryon, 1982).

Ocorrência: formações Solimões (Coari: P2, P9, P11, P12 e P13) e Içá (Coari: P2).

\section{Cyathidites sp. 2}

Fig. 3.4

Descrição: esporo simples, médio, trilete, com exina psilada; radiossimétrico, âmbito triangular de lados levemente côncavos ou convexos e vértices arredondados, laesura com aproximados $3 / 4$ do raio do esporo.

Dimensões (436 espécimes): diâmetro equatorial 32(40)48 $\mu \mathrm{m}$, exina 0,5-0,7 $\mu \mathrm{m}$ de espessura.

Observações: é correspondente a Psilatriletes sp. 3 de Banerjee (1966). 0 termo Psilatriletes foi introduzido por van der Hammen (1956) para todas as formas triletes psiladas, todavia para Potonié (1960) o termo Psilatriletes é inválido. Afinidade botânica: Cyatheaceae (Cyathea). Ocorrência: formações Solimões (Coari: P2, P9, P12 e P13) e Içá (Coari: P2).

\section{Cyathidites sp. 3}

Fig. 3.2

Descrição: de esporo simples, médio, trilete, com exina psilada; radiossimétrico, âmbito triangular de lados levemente côncavos ou convexos e vértices arredondados, laesura com aproximados $3 / 4$ do raio do esporo.

Dimensões (2 espécimes): diâmetro equatorial 32 a $48 \mu \mathrm{m}$; exina 0,5 a $0,7 \mu \mathrm{m}$ de espessura.

Ocorrência: Formação Solimões (Alto Solimões: P6).

\section{Deltoidospora Miner 1935}

\section{Deltoidospora adriennis (Potonié \& Gelletich 1933) Fredericksen 1983}

Fig. 3.1

Dimensões (2380 espécimes): diâmetro equatorial $40(48) 58 \mu \mathrm{m}$, exina de 0,5 a $0,7 \mu \mathrm{m}$ de espessura.

Registros prévios: Brasil (Lima et al., 1985b; Hoorn, 1993; Leite, 2004, 2006; Latrubesse et al., 2007;

Santos et al., 2010; Silva-Caminha et al., 2010).

Afinidade botânica: Pteridaceae, Acrostichum aureum (Lorente, 1986).

Informações ecológicas: Acrostichum aureum é uma samambaia halófita associada à vegetação de mangue (Medina et al., 1990).

Ocorrência: formações Solimões (Coari: P2, P9, P12 e P13; Alto Solimões: P1, P3, P4, P5, P6, P7 e P8) e Içá (Coari: P2).

\section{Kuylisporites Potonié 1956}

\section{Kuylisporites waterbolkii Potonié 1956}

Fig. 3.5

Dimensões (126 espécimes): diâmetro equatorial: 26(32)38 $\mu \mathrm{m}$.

Registros prévios: Brasil (Regali et al., 1974ab; Hoorn, 1993, 1994a; Silva-Caminha et al., 2010) e Venezuela (Lorente, 1986).

Afinidade botânica: Cyatheaceae, Hemitelia (Lorente, 1986).

Informações ecológicas: Para Lorente (1986), este táxon corresponde a Hemitelia grandifolia, que ocorre em florestas tropicais montanhosas.

Ocorrência: Formação Solimões (Coari: P2, P9, P11, P12 e P13; Alto Solimões: P1).

\section{Foveotriletes Potonié 1956}

Foveotriletes ornatus Regali et al. 1974

Fig. 3.6

Dimensões (3 espécimes): diâmetro equatorial 32 a $34 \mu \mathrm{m}$.

Registros prévios: Brasil (Regali et al., 1974ab) e Colômbia (Jaramillo et al., 2011). 
Afinidade botânica: desconhecida.

Ocorrência: Formação Solimões (Alto Solimões: P1).

\section{Psilatriletes Potonié 1956}

Psilatriletes peruanus Hoorn 1994

Fig. 3.7

Dimensões (124 espécimes): diâmetro equatorial 40(44) $48 \mu \mathrm{m}$.

Registros prévios: Brasil, Colômbia e Peru (Hoorn, 1994ab).

Afinidade botânica: Pteridaceae, Pteris rangiferina (Hoorn, 1993).

Informações ecológicas: ocorrência desde planícies até altas altitudes, terrestres ou epilíticas, raramente fetos aquáticos (Lorente, 1986).

Ocorrência: Formação Solimões (Coari: P2, P9, P12 e P13; Alto Solimões: P3, P4, P5, P7 e P8).

\section{Cyatheacidites Cookson 1947 emend. Potonié 1956}

\section{Cyatheacidites annulatus Cookson 1947}

Fig. 3.9

Dimensões (95 espécimes): diâmetro equatorial 54(58)62 $\mu \mathrm{m}$.

Registros prévios: Brasil (Regali et al., 1974ab; Carvalho, 1996; Garcia et al., 2008), Venezuela (Lorente, 1986; Muller et al., 1987).

Afinidade botânica: Dicksoniaceae, Losophoria quadripinnata (Lorente, 1986).

Informações ecológicas: Losophoria é feto arborescente encontrado, atualmente, desde Cuba até o Chile (Hill et al., 2001).

Ocorrência: formações Solimões (Coari: P2) e Içá (Coari: P2).

Cingulatisporites Thomson emend. Potonié 1956

Cingulatisporites sp.

Fig. 3.8

Descrição: esporo trilete, simetria radial, âmbito subcircular, presença de cíngulo restrita aos lados do grão; espessura do cíngulo 5-7 $\mu \mathrm{m}$.

Dimensões (48 espécimes): diâmetro equatorial $30(36) 42 \mu \mathrm{m}$.

Registros prévios: Brasil (Carvalho, 1996) e Venezuela (Lorente, 1986).

Afinidade botânica: Pteridaceae, Pteris (Lorente, 1986).

Ocorrência: Formação Solimões (Coari: P2, P9, P12 e P13; Alto Solimões: P1, P3 e P6).

Polypodiaceiosporites Potonié 1956

Polypodiaceiosporites potoniei Kedves 1961

Fig. 3.10a-b

Dimensões (118 espécimes): diâmetro equatorial $48(55) 62 \mu \mathrm{m}$.

Registros prévios: Brasil (Regali, 1971; Regali et al., 1974a; Lima \& Salard-Cheboldaeff, 1981; Hoorn, 1993; Lima \& Melo, 1994; Yamamoto, 1995; Maizatto, 2001; Leite, 2004).

Afinidade botânica: Pteridacea, Pteris (Lorente, 1986).

Informações ecológicas: ocorre desde o nível do mar até as altas altitudes dos Andes (Lorente, 1986).

Ocorrência: formações Solimões (Coari: P2, P9, P11, P12 e P13; Alto Solimões: P3, P4, P7 e P8) e Içá (P2).

\section{Polypodiaceiosporites pseudopsilatus Lorente} 1986

Fig. 4.1a-b

Dimensões (11 espécimes): diâmetro equatorial 28(31)34 $\mu \mathrm{m}$.

Registro prévio: Venezuela (Lorente, 1986).

Afinidade botânica: Pteridaceae, Pteris rangiferina (Lorente, 1986).

Informações ecológicas: diferentes tipos de Pteris foram listados por Posthumus (1929) em áreas a montante de montanhas. Especificamente Pteris rangiferina, usada para comparação, foi coletada da Ilha de Java, Indonésia, em altitudes de 1.350 a $1.800 \mathrm{~m}$ (Lorente, 1986).

Ocorrência: formações Solimões (Coari: P2 e P9) e Içá (Coari: P2).

\section{Polypodiaceiosporites sp.}

Fig. 3.11

Descrição: esporo trilete, médio, simetria radial, âmbito triangular. Presença de verrugas entre as laesuras, com espessamento na região interárea, presença de cíngulo; espessura do cíngulo 2-4 $\mu \mathrm{m}$. Dimensões (2 espécimes): diâmetro equatorial 37 a $42 \mu \mathrm{m}$.

Afinidade botânica: Pteridaceae.

Ocorrência: Formação Solimões (Coari: P2).

Verrucatotriletes van Hoeken-Klinkenberg 1964 

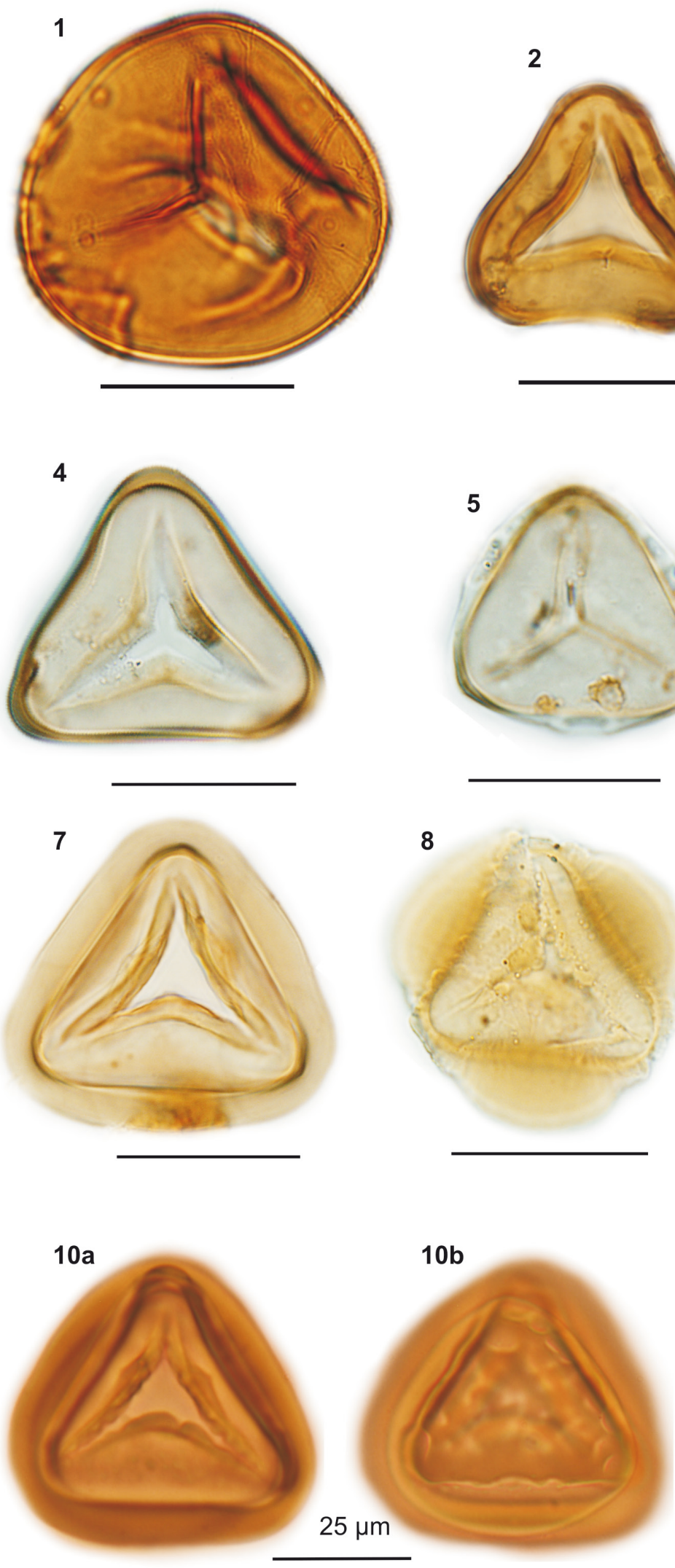

2

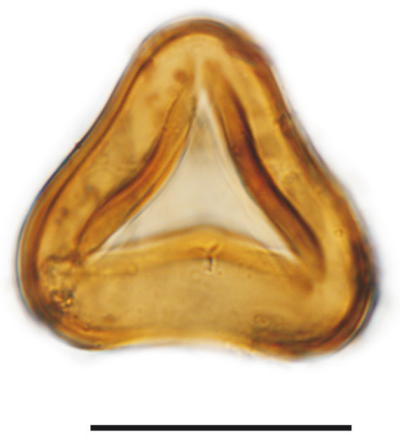

3
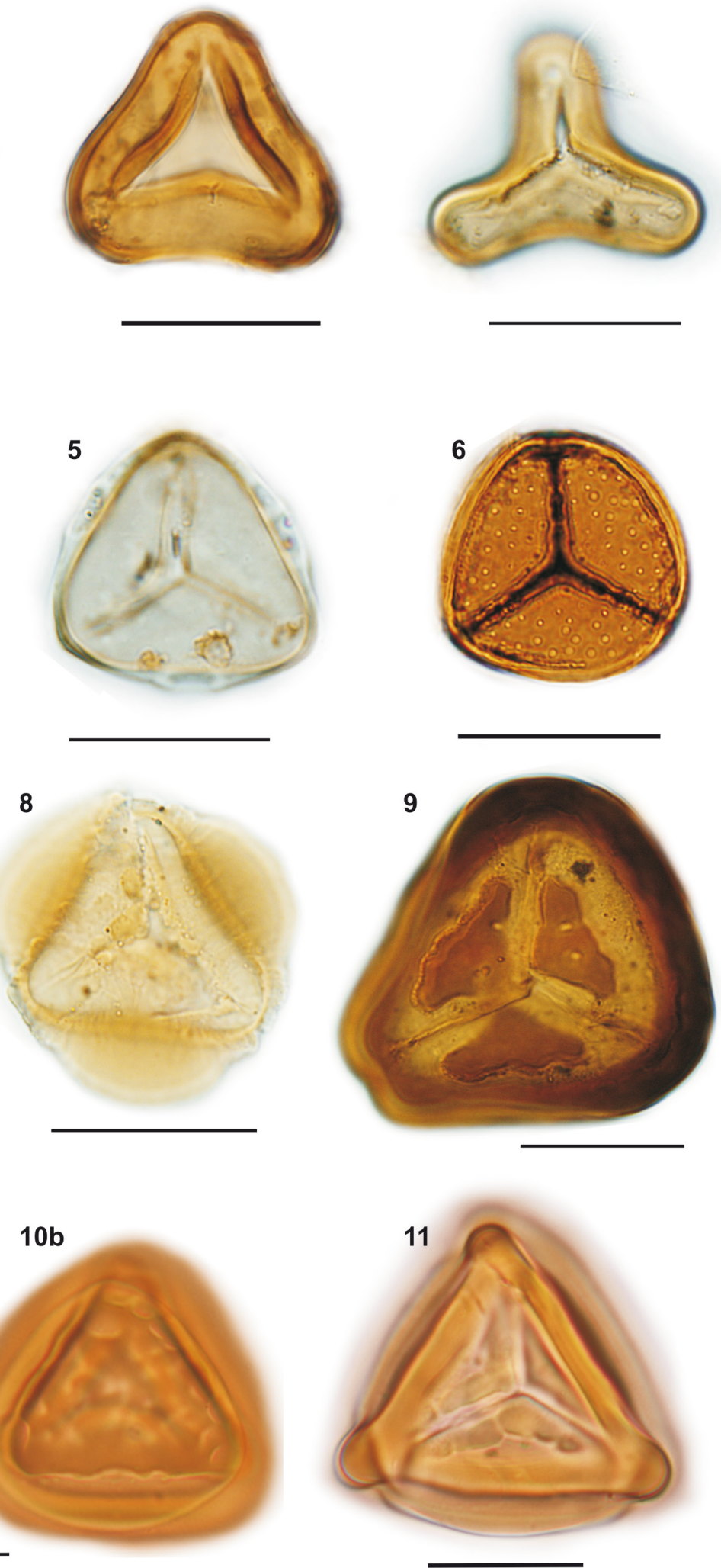

Figura 3. Fotomicrografias de palinomorfos selecionados (VP: vista polar; FP: foco proximal; FM: foco médio; FD: foco distal): 1) Deltoidospora adriennis (VP, FP, 2) Cyathidites sp. 3 (VP, FP; 3) Cyathidites sp. 1, (VP, FP; 4) Cyathidites sp. 2, (VP, FP); 5) Kuylisporites waterbolkii (VP, FM); 6) Foveotriletes ornatos (VP, FP); 7) Psilatriletes peruanus (VP, FP); 8) Cingulatisporites sp. (VP, FD); 9) Cyatheacidites annulatus (VP, FD); 10a) Polypodiaceiosporites potoniei (VP, FP); 10b) Polypodiaceiosporites potoniei (VP, FD); 11) Polypodiaceiosporites sp. (VP, FD). 
Verrucatotriletes bullatus van HoekenKlinkenberg 1964

Fig. 4.4

Dimensões (243 espécimes): diâmetro equatorial 28(35) $42 \mu \mathrm{m}$.

Registros prévios: Brasil (Hoorn, 1994b; Garcia et al., 2008).

Afinidade botânica: desconhecida.

Ocorrência: formações Solimões (Coari: P2, P9 e P12) e Içá (P2).

\section{Verrucatotriletes sp. 1}

Fig. 4.2

Descrição: esporo simples, médio, radialmente simétrico, trilete; ornamentação verrucosa, com verrugas de 1,5-2,5 $\mu \mathrm{m}$ de altura e com distintos diâmetros (1-4 $\mu \mathrm{m})$, que se distribuem em suaves ondulações; laesura quase atinge a área angular. Dimensões (594 espécimes): diâmetro equatorial 28(33)38 $\mu \mathrm{m}$.

Observação: diferencia-se de Verrucatotriletes bullatus por apresentar verrugas mais baixas e com menor uniformidade de distribuição.

Afinidade botânica: desconhecida.

Ocorrência: formações Solimões (Coari: P2, P9, P12 e P13) e Içá: (Coari: P2).

\section{Verrucatotriletes sp. 2}

Fig. 4.3

Descrição: esporo simples, grande, radialmente simétrico, trilete, verrucoso, verrugas de tamanho 1,5-2,5 $\mu \mathrm{m}$ e diâmetro de 1,5-2,5 $\mu \mathrm{m}$, uniforme; laesura com aproximamente $3 / 4$ do raio do esporo. Dimensões (617 espécimes): diâmetro equatorial 48(54)60 $\mu \mathrm{m}$.

Observações: difere de Verrucatotriletes bullatus pelo tamanho e tipos de verrugas.

Afinidade botânica: desconhecida.

Ocorrência: formações Solimões: (Coari: P2, P9, P12 e P13) e Içá: (Coari: P2).

\section{Striatriletes Potonié 1956}

Striatriletes saccolomoides Jaramillo et al. 2011 Fig. 4.5a-b

Dimensões (6 espécimes): diâmetro equatorial 34 a $40 \mu \mathrm{m}$.

Registro prévio: Colômbia (Jaramillo et al., 2011).

Afinidade botânica: Dennstaedtiaceae, Saccoloma (Jaramillo et al., 2010).

Ocorrência: Formação Solimões (Coari: P13; Alto
Solimões: P1).

Crassoretitriletes Germeraad et al. 1968

Crassoretitriletes vanraadshooveni Germeraad et al. 1968

Fig. 4.6a-b

Dimensões (599 espécimes): diâmetro equatorial 58(80)102 $\mu \mathrm{m}$.

Registros prévios: Brasil (Regali,1971; Regali et al., 1974ab, Lima \& Angulo, 1990; Carvalho, 1996; Hoorn, 1993, 1994b, 2006; Leite et al., 1997a, Leite, 2006; Silva-Caminha et al., 2010), Índia (Rao, 1995; Mandaokar \& Mukherjee, 2012), Venezuela (Germeraad et al., 1968) e Colômbia (Jaramillo et al., 2011).

Afinidade botânica: Schizaceae, Lygodium microphyllum (Hoorn, 1993).

Informações ecológicas: Lygodium microphyllum é uma samambaia trepadeira comum em pântanos e florestas úmidas do oeste da África e Indo-Malásia; também relacionada a pântanos costeiros (Hoorn, 1993).

Ocorrência: Formação Solimões (Alto Solimões: P1, P3, P4, P5, P6, P7 e P8).

\section{Magnastriatites Germeraad et al. 1968}

Magnastriatites grandiosus (Kedves \& Sole de Porta 1963) Dueñas 1980

Fig. 4.7

Dimensões (958 espécimes): diâmetro equatorial 78(89) $102 \mu \mathrm{m}$.

Registros prévios: Brasil (Regali, 1971; Hoorn, 1993; Leite, 2006; Silva-Caminha et al., 2010), Caribe e Colômbia (Germeraad et al., 1968).

Afinidade botânica: Pteridaceae, Ceratopteris (Hoorn, 1993).

Informações ecológicas: Ceratopteris é uma pequena samambaia aquática que ocorre em águas rasas de lagos, rios de planícies aluviais e pântanos costeiros (Germeraad et al., 1968; Lorente, 1986; Hoorn, 1993; Jaramillo et al., 2010).

Ocorrência: formações Solimões (Coari: P2, P9, P12 e P13; Alto Solimões: P1, P3, P4, P5, P6, P7 e P8) e Içá (Coari: P2).

Cicatricosisporites Potonié \& Gelletich 1933]

Cicatricosisporites sp.

Fig. 4.8

Descrição: esporo simples, grande, radialmente 
simétrico, anisopolar, com arredondamento nas faces e vértices, trilete, com estrias grossas (1-2 $\mu \mathrm{m}$ de altura e 2-3,5 $\mu \mathrm{m}$ de largura), mais proeminentes na região angular.

Dimensões (17 espécimes): diâmetro equatorial 72(85)98 $\mu \mathrm{m}$.

Afinidade botânica: Anemiaceae (Germeraad et al., 1968).

Ocorrência: formações Solimões (Coari: P2) e Içá (Coari: P2).

\section{Echitriletes Potonié 1956}

\section{Echitriletes muelleri Regali et al. 1974}

Fig. 4.9

Dimensões (73 espécimes): diâmetro equatorial 32(35)38 $\mu \mathrm{m}$.

Registros prévios: Brasil (Regali, 1971; Regali et al., 1974ab; Hoorn, 1993; Leite, 2004; Santos et al., 2010).

Afinidade botânica: Selaginellaceae, Selaginella (Lorente, 1986).

Ocorrência: formações Solimões (Coari: P2, P9, P11, P12 e P13; Alto Solimões: P1 e P7) e Içá (Coari: P2).

\section{Classe MONOLETES Ibrahim 1933}

Laevigatosporites Ibrahim 1933

\section{Laevigatosporites vulgaris Potonié 1934}

Fig. 5.1

Dimensões (66 espécimes): eixo longitudinal 57(64)71 $\mu \mathrm{m}$, eixo transversal 30(34)38 $\mu \mathrm{m}$.

Registro prévio: Venezuela (Rull, 2003).

Afinidade botânica: Polypodiaceae, Polypodium (Jaramillo et al., 2010).

Informações ecológicas: Polypodium é produzido por tipos distintos de samambaias (Lorente, 1986), que habitam regiões variadas; comumente encontrados em ambientes deltaicos, em áreas de mangue, sendo menos recorrente na faixa costeira. Ocorrência: formações Solimões (Coari: P2, P9, P12 e P13) e Içá (Coari: P2).

\section{Laevigatosporites sp. 1}

Fig. 5.2

Descrição: esporo simples, grande, simetria bilateral, elíptico (em vista polar), com lado distal convexo e lado proximal ligeiramente côncavo, monoletes, psilado; eixo longitudinal de proporção 1,5 maior que o eixo transversal; laesura distinta, variando de $40-70 \%$ do comprimento do esporo; exina 2-4 $\mu \mathrm{m}$ de espessura.

Dimensões (51 espécimes): maior eixo 57(72)82 $\mu \mathrm{m}$, menor eixo 29(35)41 $\mu \mathrm{m}$.

Afinidade botânica: Polypodiaceae, Polypodium.

Ocorrência: formações Solimões (Coari: P2) e Içá (Coari: P2).

\section{Laevigatosporites sp. 2}

Fig. 5.3

Descrição: esporo simples, médio, simetria bilateral, elíptico, com lado distal convexo e lado proximal ligeiramente côncavo, monoletes, psilado; eixo longitudinal de proporção 0,5 maior que o eixo transversal; laesura distinta, $65-75 \%$ do comprimento do esporo, exina 0,5-1,0 $\mu \mathrm{m}$ de espessura.

Dimensões (41 espécimes): maior eixo 20(22)24 $\mu \mathrm{m}$, menor eixo 10(12)14 $\mu \mathrm{m}$.

Afinidade botânica: Polypodiaceae (Polypodium).

Ocorrência: formações Solimões (Coari: P2, P9, P11, P12 e P13; Alto Solimões: P3) e Içá (Coari: P2).

\section{Laevigatosporites sp. 3 Fig. 5.4}

Descrição: esporo simples, pequeno, com simetria bilateral, elíptico, com lado distal convexo e lado proximal côncavo, monolete, psilado; eixo longitudinal de proporção 1,5 maior que o eixo transversal; laesura distinta, variando de $75-90 \%$ do comprimento do esporo, exina 0,5-1, $0 \mu \mathrm{m}$ de espessura.

Dimensões (87 espécimes): eixo maior 38(40)42 $\mu \mathrm{m}$, menor eixo 12(18)24 $\mu \mathrm{m}$.

Afinidade botânica: Polypodiaceae (Polypodium). Ocorrência: formações Solimões (Coari: P2, P9, P11, P12 e P13) e Içá (Coari: P2).

\section{Laevigatosporites sp. 4 \\ Fig. 5.5}

Descrição: esporo simples, médio, com simetria bilateral, elíptico, com lado distal convexo e lado proximal côncavo, monoletes, psilado; eixo longitudinal de proporção duas vezes maior que o eixo transversal; laesura distinta, variando de 65$80 \%$ do comprimento do esporo, exina $0,5-1,0 \mu \mathrm{m}$ de espessura.

Dimensões (87 espécimes): eixo maior 38(40)42 $\mu \mathrm{m}$, menor eixo $16(20) 24 \mu \mathrm{m}$.

Afinidade botânica: Polypodiaceae (Polypodium). Ocorrência: formações Solimões (Coari: P2, P9, 

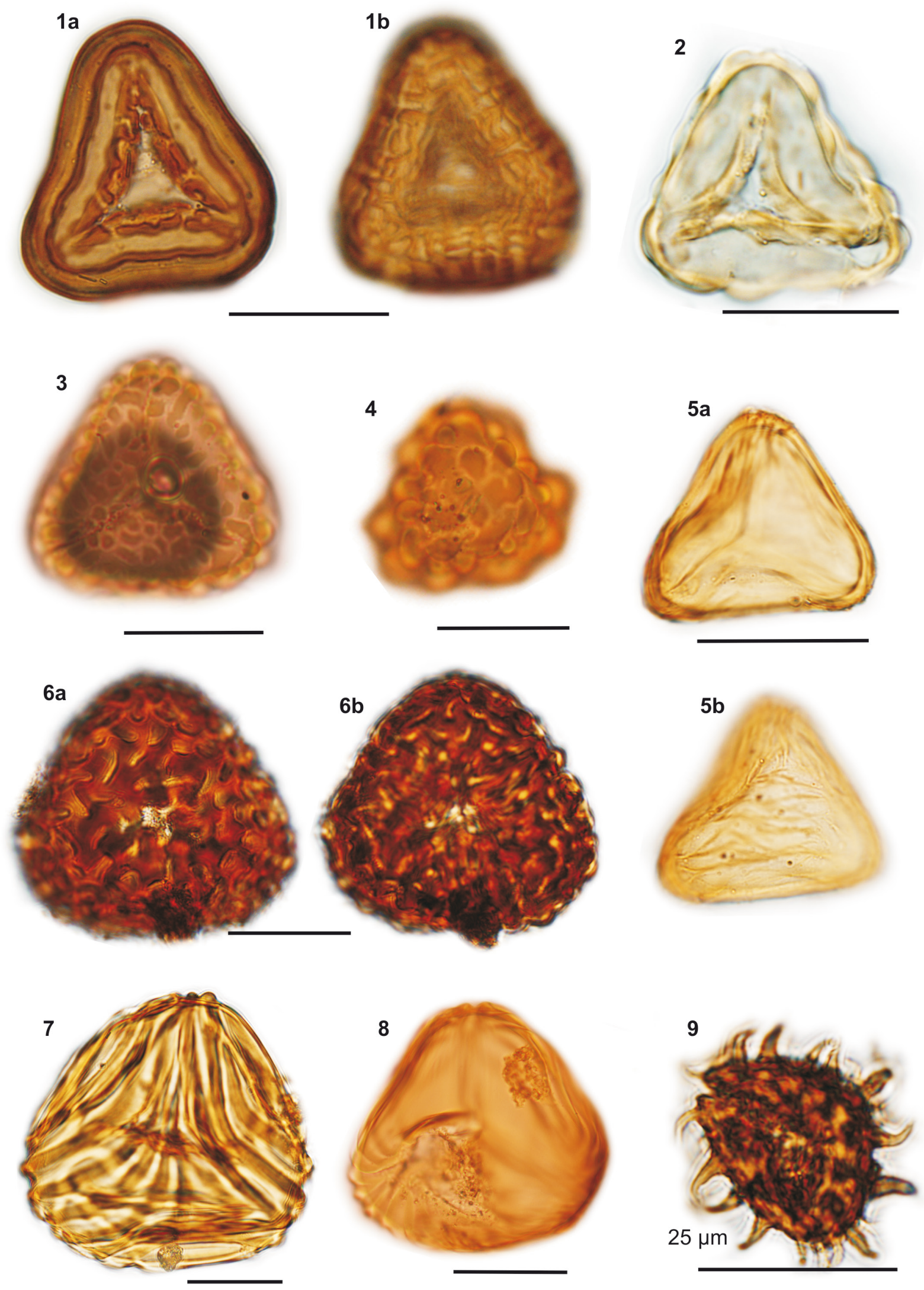

Figura 4. Fotomicrografias de palinomorfos selecionados (VP: vista polar; FP: foco proximal; FD: foco distal; FM: foco médio): 1a) Polypodiaceiosporites pseudopsilatus (VP, FP); 1b) Polypodiaceiosporites psedopsilatus (FD); 2) Verrucatotriletes sp. 1 (VP, FP); 3) Verrucatotriletes sp. 2 (VP, FP); 4) Verrucatotriletes bullatus (VP, FD); 5a) Striatriletes saccolomoides (VP, FP); 5b) Striatriletes saccolomoides (VP, FM); 6a) Crassoretitriletes vanraadshooveni (VP, FD); b) Crassoretitriletes vanraadshooveni (VP, FP); 7) Magnastriatites grandiosus (VP, FD); 8) Cicatricosisporites sp. (vista obliqua, FD); 9) Echitriletes muelleri (VP, FP). 
P11, P12 e P13) e Içá (Coari: P2).

Polypodiisporites Potonié 1956 emend. Khan \& Martin 1971

Polypodiisporites radiatus Pocknall \& Mildenhall 1984

Fig. 5.6

Dimensões (1349 espécimes): maior eixo 47(54)61 $\mu \mathrm{m}$, menor eixo 22(26)30 $\mu \mathrm{m}$.

Afinidade botânica: provavelmente Polypodiaceae. Ocorrência: Formação Solimões (Coari: P2, P9 e P12).

\section{Verrucatosporites Thomson \& Pflug 1953}

Verrucatosporites usmensis (van der Hammen 1956) Germeraad et al. 1968

Fig. 5.7

Dimensões (291 espécimes): eixo maior 48(53)58 $\mu \mathrm{m}$, eixo menor 22(26)30 $\mu \mathrm{m}$.

Registros prévios: Brasil (Regali et al., 1974ab; Lima \& Angulo, 1990; Lima et al., 1991; Hoorn, 1993, 1994ab; Santos et al., 2010), Colômbia (Jaramillo et al., 2011), Venezuela (Germeraad et al., 1968).

Afinidade botânica: Polypodiaceae, Stenochlaena palustris (Germeraad et al., 1968; Playford, 1982). Informações ecológicas: forma terrestre, raramente samambaias aquáticas, que ocorrem desde a planície à floresta de montanha.

Ocorrência: Formação Solimões (Coari: P11 e P13; Alto Solimões: P1, P3, P4, P6, P7 e P8).

Verrucatosporites favus (Potonié 1931) Thomson \& Pflug 1953

Fig. 5.8

Dimensões (751 espécimes): maior eixo 32(38)43,5 $\mu \mathrm{m}$, menor eixo 20(28)37 $\mu \mathrm{m}$.

Registro prévio: Polônia (Worobiec, 2009).

Afinidade botânica: Dennstaedtiaceae (Worobiec, 2009).

Ocorrência: formações Solimões (Coari: P2, P9, P11, P12 e P13) e Içá (Coari: P2).

\section{Verrucatosporites sp. 1}

Fig. 5.9

Descrição: esporo simples, médio, heteropolar, com simetria bilateral, monolete, verrucoso; contorno elíptico (em vista polar) e côncavoconvexo (em vista equatorial), com verrugas robustas, irregularmente distribuídas por toda a superfície do grão, com diminuição no tamanho em direção ao polo proximal.

Dimensões (104 espécimes): maior eixo 32(40)48 $\mu \mathrm{m}$, menor eixo 16(20)24 $\mu \mathrm{m}$.

Afinidade botânica: Polypodiaceae.

Ocorrência: formações Solimões (Coari: P2, P9 e P12) e Içá (Coari: P2).

\section{Verrucatosporites sp. 2}

Fig. 5.10

Descrição: esporo simples, médio, heteropolar, com simetria bilateral, monoletes, verrucoso. Elíptico (em vista polar) e côncavo-convexo (em vista equatorial); verrugas pequenas, 1,5-2,5 $\mu \mathrm{m}$ de altura e diâmetro, uniformemente distribuídas. Dimensões (18 espécimes): maior eixo 36(42)48 $\mu \mathrm{m}$, menor eixo $18(21) 24 \mu \mathrm{m}$.

Afinidade botânica: Polypodiaceae.

Ocorrência: formações Solimões (Coari: P2, P9 e P12) e Içá (Coari: P2).

\section{Perinomonoletes Krutzsch 1967}

Perinomonoletes sp.

Fig. 5.11

Dimensões (17 espécimes): maior eixo 33(35)38 $\mu \mathrm{m}$, menor eixo 16(18)20 $\mu \mathrm{m}$.

Registros prévios: Brasil (Hoorn, 1993; Leite, 2004, 2006) e Venezuela (Rull, 2001).

Afinidade botânica: Aspleniaceae, Asplenium ou Thelypteraceae, Thelypteris (Hoorn, 1993).

Ocorrência: formações Solimões (Coari: P2, P9, P11, P12 e P13) e Içá (Coari: P2).

\subsection{Grãos de pólen de gimnospermas}

Classe VESICULATAE Iversen \& Troels-Smith 1950

Podocarpidites Cookson 1947 ex Couper 1953

Podocarpidites sp. 1

Fig. 5.12

Descrição: grão de pólen mônade, vesiculado (bissacado), grande, heteropolar, com simetria bilateral, corpo central aproximadamente esferoidal com leptoma no polo distal; sacos aéreos com retículo de malhas irregulares.

Dimensões (83 espécimes): comprimento total (incluindo os sacos) de 65(75)85 $\mu \mathrm{m}$; diâmetro do corpo central 18(27)36 $\mu \mathrm{m}$; altura dos sacos $47(48) 49 \mu \mathrm{m}$. 
Afinidade botânica: Podocarpaceae, Podocarpus (Hoorn, 1993).

Informações ecológicas: constituem árvores e arbustos comuns na região andina, mas em território brasileiro é um componente da mata de Araucaria, bem como ocorrente na mata atlântica. Ocorrência: Formação Solimões (Coari: P9, P11, P12 e P13; Alto Solimões: P1).

\section{Podocarpidites sp. 2}

Fig. 5.13

Descrição: grão de pólen mônade, vesiculado (bissacado), médio, heteropolar, com simetria bilateral, corpo central aproximadamente esferoidal com leptoma no polo distal; sacos aéreos com retículo de malhas irregulares.

Dimensões (25 espécimes): comprimento total (incluindo os sacos) de 64(70)76 $\mu \mathrm{m}$; diâmetro do corpo central $16(22) 28 \mu \mathrm{m}$; altura dos sacos 26(31)36 $\mu \mathrm{m}$.

Afinidade botânica: Podocarpaceae (Podocarpus). Ocorrência: Formação Solimões (Alto Solimões: P1, P3 e P7).

\subsection{Algas clorofíceas}

\section{Divisão CHLOROPHYTAE} Classe CHLOROPHYCEAE

\section{Pediastrum Meyen 1829}

\section{Pediastrum sp. 1}

Fig. 6.1

Descrição: cenóbio compacto, com células unidas umas às outras, formando anel com 8 células simples.

Dimensões (217 espécimes): diâmetro total 62-82 $\mu \mathrm{m}$, larguras das células 4,5-5,5 $\mu \mathrm{m}$ e comprimento do processo 15-22 $\mu \mathrm{m}$.

Afinidade botânica: Ordem Chlorococcales, Família Hydrodictyaceae (Meyen, 1829).

Ocorrência: Formação Solimões (Alto Solimões: P3, P7).

Observação: todos os espécimes relacionados ao gênero Pediastrum apresentadas aqui, foram verificadas utilizando microscopia óptica sob a luz fluorescente.

\section{Pediastrum sp. 2}

Fig. 6.2

Descrição: cenóbio compacto, com células inteiramente unidas umas às outras, formando um anel com 6 células e unidas a duas células centrais, as células são bifurcadas no anel e com quatro pontas nas células centrais.

Dimensões (82 espécimes): diâmetro total 35-42 $\mu \mathrm{m}$, largura das células 5-6 $\mu \mathrm{m}$ e comprimento do processo 5-6 $\mu \mathrm{m}$.

Ocorrência: Formação Solimões (Coari: P2, P9, P12 e P13; Alto Solimões: P3 e P7).

\section{Pediastrum sp. 3}

Fig. 6.3

Descrição: cenóbio compacto, com células inteiramente unidas umas às outras, formando 2 anéis, com 5 células no primeiro anel, e 10 células no segundo, sendo unidas a uma célula central, as células são bifurcadas no segundo anel, com quatro pontas no primeiro anel.

Dimensões (32 espécimes): diâmetro total 40-45 $\mu \mathrm{m}$, largura das células $3-5,5 \mu \mathrm{m}$ e comprimento do processo 3-5,5 $\mu \mathrm{m}$.

Ocorrência: Formação Solimões (Alto Solimões: P3 e P7).

\section{Pediastrum sp. 4}

Fig. 6.4

Descrição: cenóbio tipo compacto, as células são inteiramente unidas umas às outras, formando 3 anéis, com 6 células no primeiro anel, 8 células no segundo, 22 células no terceiro, sendo unidas a 2 células centrais; as células são bifurcadas no anel segundo anel, com 4 pontas no primeiro anel.

Dimensões (4 espécimes): diâmetro total 45-50 $\mu \mathrm{m}$, largura das células $2,5-3,5 \mu \mathrm{m}$ e comprimento do processo $2,5-3,5 \mu \mathrm{m}$.

Ocorrência: Formação Solimões (Alto Solimões: P3 e P7).

\section{Pseudoschizaea Thiergart \& Frantz 1962}

\section{Pseudoschizaea circulus Christopher 1976}

Fig. 6.5

Dimensões (74 espécimes): diâmetro de 38(40)42 $\mu \mathrm{m}$.

Afinidade biológica: cisto de alga de água doce, relacionada à Zygnemataceae, mas podem apresentar relação com as algas Oedogoniaceae (Scaphati et al., 2009). 0 gênero-forma ocorre ocasionalmente em sedimentos de águas rasas subtropicais (Davis, 1992). Para Carrión \& Navarro (2002), os cistos de Pseudoschizaea habitam zonas climáticas relativamente quentes com fases de dessecação ou maior seca de verão (secagens 

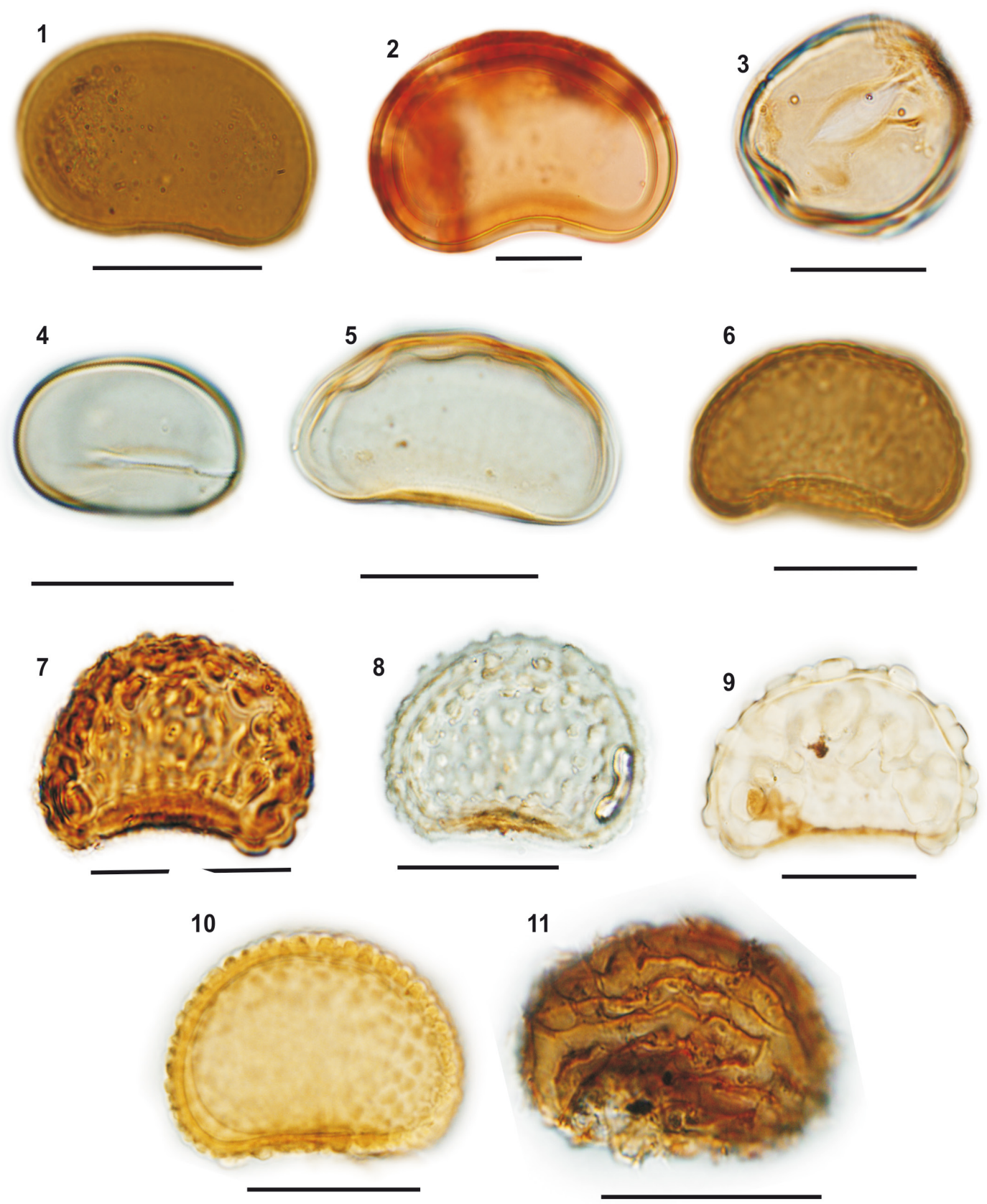

12
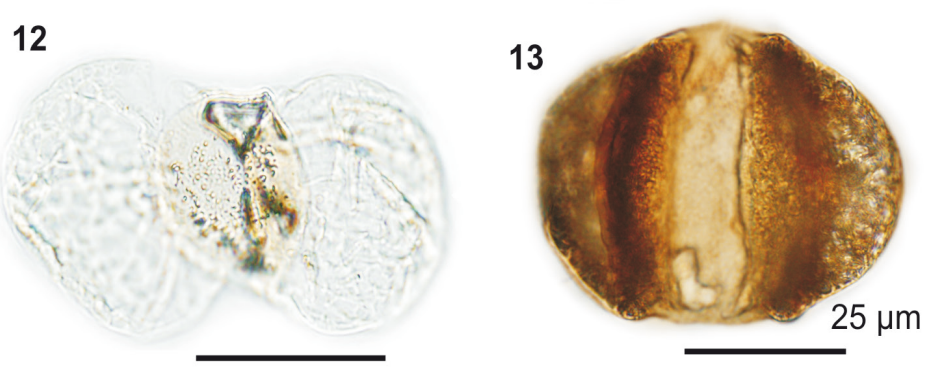

13 $25 \mu \mathrm{m}$

Figura 5. Fotomicrografias de palinomorfos selecionados (VE: vista equatorial; FD: foco distal; FP: foco proximal): 1) Laevigatosporites vulgaris VE, FD; 2) Laevigatosporites sp. 1, VE, FP; 3) Laevigatosporites sp. 2, VP, FD; 4) Laevigatosporites sp. 3, VP, FD; 5) Laevigatosporites sp. 4, VE, FD; 6) Polypodiisporites radiatus, VE, FP; 7) Verrucatosporites usmensis, VE, FD; 8) Verrucatosporites favus, VE, FD; 9) Verrucatosporites sp. 1, VE, FD; 10) Verrucatosporites sp. 2, VE, FP; 11) Perimonoletes sp., VE, FD; 12) Podocarpidites sp. 1, VP, FD; 13) Podocarpidites sp. 2, VP, FD. 
sazonais locais).

Ocorrência: Formação Solimões (Coari: P2, P9, P12 e P13).

\section{Pseudoschizaea sp.}

Fig. 6.6

Descrição: formas circulares a ovais em vista polar, biconvexas em vista equatorial, inaperturadas; cada hemisfério com séries de 5 a 12 estrias ao redor da área polar, concêntrica, formam anéis, que podem ser completos, incompletos, bifurcados ou em arranjo em espiral.

Dimensões (212 espécimes): diâmetro de 42(50)58 $\mu \mathrm{m}$.

Afinidade biológica: cisto de alga de água doce, relacionada à Zygnemataceae, mas podem apresentar relação com as algas Oedogoniaceae (Scaphati et al., 2009).

Ocorrência: Formação Solimões (Coari: P2, P9, P12 e P13).

\section{Ovoidites Potonié 1951}

\section{Ovoidites sp.}

Fig. 6.7

Descrição: esporo elíptico em vista equatorial, rompido em duas partes iguais no equador; parede psilada, com $0,5 \mu \mathrm{m}$ de espessura.

Dimensões (148 espécimes): diâmetro equatorial 20-35 $\mu \mathrm{m}$, eixo polar 24-28 $\mu \mathrm{m}$.

Afinidade biológica: segundo van Geel \& van der Hammen (1978), tanto Ovoidites quanto Schizosporis representam esporos de Zygnemataceae relacionados com o gênero atual Spirogyra.

Ocorrência: Formação Solimões (Coari: P2, P9, P11, P12 e P13).

\subsection{Alga dinofícea}

Divisão DINOFLAGELATA (Bütschli, 1885)

Fensome et al. 1993

Classe DINOPHYCEAE Pascher 1914

Diphyes Cookson 1965

Diphyes sp.

Fig. 6.9

Dimensões (1 espécime): diâmetro do corpo central de $45 \mu \mathrm{m}$ x $52 \mu \mathrm{m}$. Comprimento do processo antapical $18 \mu \mathrm{m}$, largura do processo antapical $9 \mu \mathrm{m}$.
Ocorrência: Formação Solimões (Alto Solimões: P7).

\subsection{Fragmento de invertebrado}

Escolecodonte indeterminado

Fig. 6.8

Descrição: representado por um conjunto de mandíbula inferior e superior, robusta, com estrutura dentiforme, variando de 3 a 4 dentes.

Dimensões (1 espécime): mandíbula 47,5 $\mu \mathrm{m}$, dentes de tamanho variando de 2,5 a $3,5 \mu \mathrm{m}$.

Afinidade biológica: correspondem a estruturas mandibulares de anelídeos poliquetas (Armstrong \& Brasier, 2005), porém podem estar relacionadas a rádulas de gastrópodes (Calvo, 1987).

Ocorrência: Formação Solimões (Coari: P2).

\section{Discussão dos resultados}

Os dados taxonômicos apresentados são complementados com a distribuição estratigráfica por táxon e afloramento, constante no quadro 1, bem como com suas representações quantitativas, ainda inéditas (segundo Silveira, 2015), a serem utilizados para fins bioestratigráficos e de interpretação paleoambiental em trabalhos conseguintes.

Além de suas características estratigráficas (Nogueira et al., 2013), as formações Solimões e Içá se distinguem pela ocorrência, na última, de Alnipollenites verus, espécie ali observada por Silveira (2015) e Silveira \& Souza (no prelo). A unidade apresenta-se distribuída apenas na região de Coari, sendo reconhecida exclusivamente no P2. Ainda que na região de Coari a Formação Solimões apresente mesma composição palinológica que a Formação Içá, as abundâncias desses palinomorfos são distintas.

De forma geral, os depósitos da Formação Solimões apresentam maior potencial à preservação dos palinomorfos. Na região de Coari, apesar de serem registrados vários espécimes em tétrade, muitos dos esporos de pteridófitas se apresentam corroídos e/ou rasgados. Já na região do Alto Solimões os palinomorfos apresentam excelente estado de preservação.

Dentre os táxons analisados nas duas áreas de estudo, predominam representantes dos grupos continentais. Os esporos de fungos da região de Coari apresentam maior diversificação, particularmente oriundos da Formação Solimões, representados por Monoporisporites sp. 1, 2 e 3, Foveodiporites sp., Fusiformisporites crabbii, Mediaverrusporonites elsikii, Meliolinites sp., Quilonia 


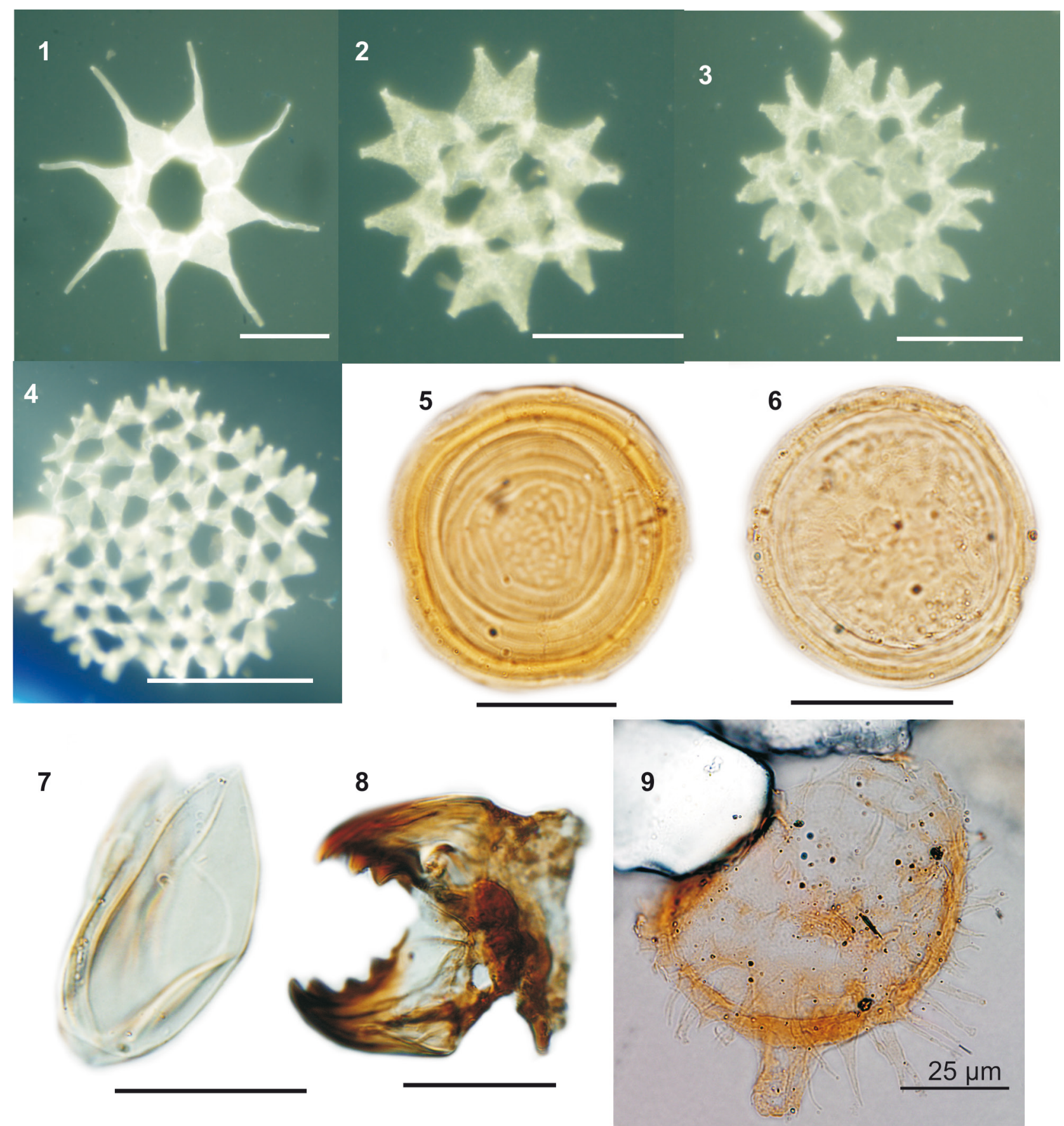

Figura 6. Fotomicrografias de palinomorfos selecionados (1-4 obtidas sob luz de fluorescência): 1) Pediastrum sp. 1; 2) Pediastrum sp. 2; 3) Pediastrum sp. 3; 4) Pediastrum sp. 4; 5) Pseudoschizaea circulus; 6) Pseudoschizaea sp.; 7) Ovoidites sp.; 8) Escolecodonte indeterminado; 9) Diphyes sp.

sp. Além dessas formas, na Formação Içá ocorrem também Diporicellaesporites sp. 1 e Diporicellaesporites sp. 2.

Na região do Alto Solimões ocorre menor diversidade de pteridófitas, contudo determinadas formas como Deltoidospora adriennis, Crassoretitriletes vanraadshooveni e Magnastriatites grandiosus são verificadas em maior abundância que em qualquer outro afloramento da região de Coari.

Entre os elementos aquáticos, as algas clorofíceas como Pediastrum sp. 2, Ovoidites sp., Pseudoschizeae circulus e Pseudoschizeae sp. se distribuem em depósitos das formações Içá e Solimões na região de Coari, porém com poucos espécimes. $\mathrm{Na}$ região do Alto Solimões o gênero Pediastrum spp. é registrado em grande abundância, além de um único espécime de cisto de dinoflagelado relacionado ao gênero Diphyes. Segundo Matsuoka (1974), a espécie $D$. latiusculus, muito semelhante à da Bacia do Solimões, ocorre em ambiente marinho. $\mathrm{Na}$ região do Alto Solimões esta influência é pontual; a confirmação da presença da espécie corrobora os estudos de Hoorn (1993, 1994b); Leite (2006) e Hoorn et al. (2010) sobre as incursões marinhas 
miocenas e pliocenas na área. Entretanto, o registro de apenas uma espécie requer cautela para interpretações paleoambientais mais seguras.

Para os depósitos da região de Coari, foi registrado um escolecodonte na Formação Solimões. Este tipo de palinomorfo é comum em depósitos marinhos e continentais do Paleogeno (Lima \& Dino, 1984; Lima \& Amador, 1985; Lima et al., 1994, Premaor et al., 2010; Fischer et al., 2013).

\section{Conclusões}

Conjuntos palinológicos bem preservados, abundantes e diversificados são descritos para depósitos cenozoicos da Bacia do Solimões, particularmente para as formações Solimões e Içá, com documentação fotomicrográfica, com a presença de esporos (de fungos e pteridófitas), grãos de pólen de gimnospermas, cistos e colônias de algas e um escolecodonte, ampliando o conhecimento paleontológico para a região, que vem sendo alvo de novos estudos nos últimos anos.

Para a região de Coari foi constatado o predomínio de esporos de pteridófitas (aquáticas e terrestres), representadas por Polypodiaceae, Cyatheaceae e Pteridaceae, relacionadas a condições de alta umidade, durante todo o intervalo de deposição das formações Solimões e Içá, o que é corroborado pela diversificada e abundante representação de esporos de fungo. Não foi registrado qualquer indício de influência marinha para a área.

Na região de Alto Solimões a microflora compreende predominantemente esporomorfos, associados a algas, particularmente Pediastrum em grande número e diversificadas formas, que sugere deposição em lagoas de água doce, lagos e/ou rios lentos, possivelmente em condições eutróficas. A presença de um único registro de cisto de dinoflagelado indica possibilidade de influência marinha, mas requer melhor detalhamento taxonômico, bem como novas prospecções na área.

Agradecimentos -Os autores agradecem a FAPEAM e ao $\mathrm{CNPq}$ pelo financiamento das atividades de campo bem como pela concessão de bolsa de doutorado e de produtividade em pesquisa (Processos CNPq 142102/2010-5 e 308844/2010-6), aos revisores, pelas significativas contribuições. Este trabalho é parte da Tese de Doutoramento da primeira autora desenvolvida junto ao Programa de Pós-graduação em Geociências da Universidade Federal do Rio Grande do Sul.

\section{Referências}

Armstrong, H.A. \& Brasier, M.D. 2005. Microfossils. 2a. ed., Malden, Blackwell Publishing, 296 p.
Banerjee, D. 1966. A note on Tertiary microflora from Andaman Islands, India. Polen et Spores, 61: 205212.

Calvo, I.S. 1987. Rádulas de gastrópodes marinhos brasileiros. Rio Grande, Editora da Fundação Universidade do Rio Grande, 201p.

Caputo, M.V. 1984. Stratigraphy, Tectonics, Paleoclimatology and Paleogeography of Nortern Basin of Brazil. Santa Barbara, 583p.Tese de Doutorado, University of California.

Carrión, J.S. \& Navarro, C. 2002. Cryptogam spores and other non-pollen microfossils as sources of palaeoecological information: case- studies from Spain. Annals Botanical Fennici, 39: 1-14.

Carvalho, M.A. 1996. Estudo paleoecológico e paleoclimático, com base em palinologia, aplicado em sedimentos pleistocênicos e pliocênicos da Bacia da Foz do Amazonas. Rio de Janeiro, 146p. Dissertação de Mestrado, Programa de Pós-Graduação em Geologia, Instituto de Geociências, Universidade Federal do Rio de Janeiro.

Cruz, N.M.C. 1984. Palinologia do linhito do Solimões no Estado do Amazonas. In: SIMPÓSIO DE GEOLOGIA DA AMAZÔNIA, 2, 1984, Manaus. Resumos expandidos, p. 473-480.

Daemon, R.F. \& Contreiras, C.J.A. 1971. Zoneamento palinológico da bacia do Amazonas. In: CONGRESSO BRASILEIRO DE GEOLOGIA, 25, 1971, São Paulo. Anais.., 3: 79-91.

Davis, O.K. 1992. Rapid climatic change in coastal southern California inferred from Pollen Analysis of San Joaquin Marsh. Quaternary Research, 37: 89-100.

Eiras, J.F., Becker, C.R., Souza, E.M., Gonzaga, F.G., da Silva, J.G.F., Daniel, L.M.F., Matsuda, N.S. \& Feijó, F.J. 1994. Bacia do Solimões. Boletim de Geociências da Petrobrás, 8(1): 17-45.

Fischer. T.V., Souza, P.A., Escamilla, J.H. \& Arai, M. 2013. Associações palinológicas do Paleógeno da Bacia de Pelotas (Poço BP-1, Brasil) e seu significado estratigráfico. Geociências, 32(4): 677-695.

Garcia, M.J., Santos, M. \& Hasui, Y. 2000. Palinologia da parte aflorante da Formação Entre-Córregos, Bacia de Aiuruoca, Terciário do Estado de Minas Gerais, Brasil. Revista Universidade Guarulhos, Geociências, Ano V (n. esp.): 259.

Garcia, M.J., Bernardes-de-Oliveira, M.E., Santos, M.A., Dino, R., Antonioli, L., Bistrichi, C.A. \& Casado, F.C. 2007. Floras neógenas Sul-americanas no contexto mundial. In: Carvalho, I.S. et al. (Eds.) Paleontologia: Cenários de Vida, vol. 1, p. 657-687.

Garcia, M.J., Bistrichi, C.A., Saad, A.R., Campanha, V.A. \& Oliveira, P.E. 2008. Stratigraphy and Paleoenvironmets of the Tanque Basin, Southeastern Brazil. Revista Brasileira de Paleontologia, 11(3): 147-168.

Garcia, M. J., Lima, F.M., Fernandes, L.A., Melo, M. S., Dino, R., Antonioli, L. \& Menezes, J.B. 2013. Idade e palinologia da Formação Guabirotuba, Bacia de Curitiba, PR, Brasil. In: CONGRESSO BRASILEIRO DE PALEONTOLOGIA, 23, 2013, Gramado. Boletim de Resumos, p. 125.

Germeraad, J.H., Hopping, C.A. \& Muller, J. 1968. Paly- 
nology of tertiary sediments from the tropical areas. Review of Palaeobotany and Palynology, 6: 189-348.

Guimarães, J.T.F., Nogueira, A.C.R., Silva Jr, J.B.C., Soares, J.L. \& Silveira, R.R. 2013. Fossil Fungi from Miocene sedimentary of the Central and Coastal Amazon Region, North Brazil. Journal of Paleontology, 87(3): 484-492.

Hill, R.S., Macphail, M.K. \& Jordan, G.J. 2001. Macrofossils associated with the fossil fern spore Cyatheacidites annulatus and their significance for Southern hemisphere biogeography. Review of Palaeobotany and Palynology, 116: 195-202.

Hoorn, C. 1993. Marine incursions and the influence of Andean tectonics on the Miocene deposital history of northwestern Amazonia: results of a palynostratigraphic study. Palaogeography, Palaeoclimatology, Palaeoecology, 105: 277-309.

Hoorn, C. 1994a. Fluvial palaeoenvironments in the intracratonic Amazonas Basin (Early Miocene - early Middle Miocene, Colombia). Palaeogeography, $\mathrm{Pa}$ laeoclimatology, Palaeoecology, 109: 1-54.

Hoorn, C. 1994b. An environmental reconstruction of the palaeo-Amazon River system (Middle-Late Miocene, NW Amazonia). Palaeogeography, Palaeoclimatology, Palaeoecology, 112: 187-238.

Hoorn, C. 2006. Mangrove forests and marine incursions in Neogene Amazonia (Lower Apaporis River, Colombia). Palaios, 21:197-209.

Hoorn, C., Wesselingh, F.P., Steage, H., Bermudez. M.A., Mora, A., Sevink, J., San Martín, I., Sanchez-Meseguer, A., Anderson, C.L., Figueiredo. J.P., Jaramillo, C., Riff, D., Negri, F.R., Hooghiemstra, H., Lundberg, J., Stadler, T., Särkinen, T. \& Antonelli, A. 2010. Amazon trough time: Andean uplift, climate change, landscape evolution, and biodiversity. Science, 330: 927-931.

Jaramillo, C., Hoorn, C., Silva, S.A.F., Leite, F., Herrera, F., Quiroz, L., Dino, R. \& Antoniolli, L. 2010. The origin of the modern Amazon Rainforest: implications of the palynological and palaeobotanical record. In: Hoorn, C. \& Wesselingh, F. P. (Ed.), Amazonia, Landscape and species Evolutions: A Look in to the past. Wiley, Wiley-Blackwell, p. 317-334.

Jaramillo, C. A., Rueda, M. \& Torres, V. 2011. A palynological zonation for the Cenozoic of the Llanos and Llanos Foothills of Colombia, Palynology, 35: 46-84.

Kalgutkar, R.M. \& Jansonius, J. 2000. Synopsis of Fossil Fungal Spores, Mycelia, and Frutifications. American Association of Stratigraphy Palynologists Foundation, Contributions Series 39, 423p.

Kalgutkar, R. M. \& Sigler, L. 1995. Some fungal form-taxa from the Maastrichtian and Palaeogene ages. Мyсоlogical Research, 99: 513-522.

Latrubesse, E.M., Silva, S.A.F., Cozzuol, M. \& Absy, M.L. 2007. Late Miocene continental sedimentation in southwestern Amazonia and its regional significance: Biotic and geological evidence. Journal of South American Earth Sciences, 23: 61-80.

Leite, F.P.R. 2004. Palinologia. In: Rossetti, D.F. \& Góes, A.M. (Ed.), O Neógeno da Amazônia Oriental. Belém, Editora do Museu Emilio Goeldi, p. 55-90.

Leite, F.P.R. 2006. Palinologia da Formação Solimões,
Neógeno da Bacia do Solimões, Estado do Amazonas, Brasil: Implicações Paleoambientais e Bioestratigráficas. Brasília, 128p. Tese de Doutorado, Programa de Pós-graduação em Geociências Aplicadas, Instituto de Geociências, Universidade de Brasília.

Leite, F.P.R., Oliveira, M.E.B., De Oliveira, P.E., Silvestre-Capelato, M.S., Arai, M. \& Trunckenbroudt, W. 1997a. Palinoflora Miocenas da Formação Pirabas e Grupo Barreiras, na Região Bragantina, Estado do Pará, Brasil. Revista da Universidade Guarulhos, Geociências, 2: 128-140.

Leite, F.P.R., Oliveira, M.E.B., Arai, M. \& Trunckenbroudt, W. 1997b. Palinoestratigrafia da Formação Pirabas e Grupo Barreiras, Mioceno do Nordeste do Estado do Pará, Brasil. Revista da Universidade Guarulhos, Geociências, 2: 141-147.

Lima, M.R. \& Amador, E.S. 1985. Análise palinológica de sedimentos da Formação Resende, Terciário do Estado do Rio de Janeiro, Brasil. In: CONGRESSO BRASILEIRO DE PALEONTOLOGIA, 8, 1983, Rio de Janeiro. Coletânea de trabalhos paleontológico, Rio de Janeiro, MME-DNPM, p. 371-378.

Lima, M.R. \& Angulo, R.J. 1990. Descoberta de microflora em um nível linhítico da Formação Alexandra, Terciário do Estado do Paraná, Brasil. Anais da Academica Brasileira de Ciências, 62(4): 357-371.

Lima, M.R. \& Cunha, F.L.S. 1986. Análise palinológica de um nível linhítico da Bacia de São José de Itaboraí, Terciário do Estado do Rio de Janeiro, Brasil. Anais da Academia Brasileira de Ciências, 58(4): 579-588.

Lima, M.R. \& Dino, R. 1984. Palinologia de Amostras da Bacia de Bonfim, Terciário do Estado de São Paulo, Brasil. Boletim do Instituto de Geociências USP, 15: 1-11.

Lima, M.R. \& Melo, M.S. 1994. Palinologia de depósitos rudáceos da região de Itatiaia, bacia de Resende, Rio de Janeiro. Geonomos, 2(1): 12-21.

Lima, M.R. \& Salard-Cheboldaeff, M. 1981. Palynologie des bassins de Gandarela et Fonseca (Eocene de L'etat de Minas Gerais, Brésil). Boletim do Instituto de Geociências USP, 12: 33-54.

Lima, M.R., Salard-Cheboldaeff, M. \& Suguio, K. 1985a. Étude Palynologique de la Formation Tremembé, Tertiarie du Bassin de Taubaté, (Etat de São Paulo, Brésil), d'après les Echantillons du Sondage $n^{\circ} .42$ du CNP. In: Coletânea de trabalhos paleontológicos, Brasília, DNPM. Série Geologia, 27, Seção Paleontologia e Estratigrafia, 2, p. 379-393.

Lima, M.R., Vespucci, J.B.O. \& Suguio, K. 1985b. Estudo palinológico de uma camada de linhito da Formação Caçapava, Bacia de Taubaté, Terciário do Estado de São Paulo, Brasil. Anais da Academia Brasileira de Ciências, 57: 183-197.

Lima, M.R., Melo, M.S. \& Coimbra, A.M. 1991. Palinologia de Sedimentos da Bacia de São Paulo, Brasil. Revista do Instituto Geológico, 12: 7-20.

Lima, M. R., Riccomini, C. \& Souza, P.A. 1994. Palinologia de folhelhos do Gráben de Casa de Pedra, Terciário do Estado do Rio de Janeiro, Brasil. Acta Geológica Leopoldensia, 39(2): 485-504.

Lima, M. R., Cabral Jr., M. \& Stefani, F.L. 1996. Palinologia 
de sedimentos da Formação Macacu-Rifte da Guanabara, Terciário do Estado do Rio de Janeiro. Anais da Academia Brasileira de Ciências, 68(4): 531-543.

Lorente, M.A. 1986. Palynology and palynofacies of upper Tertiary in Venezuela. Dissertationes Botanicae, 99: $222 \mathrm{p}$.

Maia, R.G.N., Godoy, H.K., Yamaguti, H.S., Moura, P.H., Costa, F.S.F. da, Holanda, M.A. \& Costa, J.A. 1977. Projeto Carvão no Alto Solimões, Manaus, DNPM/CPRM, $137 \mathrm{p}$.

Maizatto, J.R. 2001. Análise bioestratigráfica, paleoecológica e sedimentológica das bacias terciárias do Gandarela e Fonseca - Quadrilátero Ferrífero - Minas Gerais, com base nos aspectos palinológicos $e$ sedimentares. Ouro Preto, 249p. Tese de Doutorado, Programa de Pós-graduação em Evolução Crustal e Recursos Naturais, Escola de Minas, Universidade Federal de Ouro Preto.

Mandaokar, B.D. \& Mukherjee, D. 2012. Palynological investigation of Early Miocene sediments exposed at panruti, cuddalore district, Tamil Nadu, India. International Journal of Geology, Earth and Environmental Science, 2(3): 157-175.

Matsuoka, K. 1974. Some plant microfossils from the Miocene Fujiwara Group, Nara, Central Japan. The Palaeontological Society of Japan, Transactions and Proceeding, New Series, 94: 319-340.

Medina, E.E. Cuevas, M.P. \& Lugo, A. 1990. Soil salinity, sun exposure, and growth of Acrostichum aureum, the mangrove fern. Botanical Gazette, 151(1): 41-45.

Melo, A.F.F. \& Villas Boas, P.F. 1993. Caracterização e Distribuição da Formação Içá na Porção Noroeste do Estado do Amazonas. In: SIMPÓSIO DE GEOLOGIA DA AMAZÔNIA, 4, 1993, Manaus. Resumos Expandidos, p. 210-211.

Mercês, M.B.F. 2005. Palinologia do Jazigo Quiririm, Formação Tremembé, Bacia de Taubaté: Implicações Palinocronoestratigráficas, Paleovegetacionais e Paleoclimáticas. Guarulhos, 81p. Trabalho de Conclusão de Curso, Curso de Ciências Biológicas, Universidade Guarulhos.

Mercês, M.F., Garcia, M.J. \& Bernardes-de-Oliveira, M.E.C. 2006. A paleopalinologia do jazigo Quiririm, Formação Tremembé, Bacia de Taubaté: implicações paleoambientais. In: SIMPÓSIO DO CRETACEO DO BRASIL, 7, e SIMPÓSIO DO TERCIÁRIO DO BRASIL, 1, 2006, Serra Negra. Boletim de Resumos..., p. 85.

Meyen, F.J.F. 1829. Beobachtungen Über Einige Niedere Algenformen. Nova Acta Physico-Medica Academiae Caesceae Leopoldino-Carolinae Naturae, 14: 768778.

Muller, J., Giacomo, E. \& van Erve, A.W. 1987. A palynological zonation for the Cretaceous, Tertiary and Quaternary of Northern South America. American Association of Stratigraphy Palynologists Foundation, Contributions Series 19, p 7-76.

Nogueira, A.C.R., Silveira, R.R. \& Guimarães, J.T.F. 2013. Neogene-Quaternary sedimentar and paleovegetation history of eartern Solimões Basin, central Amazon region. Jornal of South American Earth Sciences, 46: 89-99.
Playford, G. 1982. Neogene palynomorphs from the Huon Peninsula, Papua New Guinea. Palynology, 6: 29-54.

Posthumus, 0. 1929. The ferns of Surinam and of French and British Guaina. Java, Malang, 196p.

Potonié, H. 1893. Die flora des Rotliegenden von Thüringen. Abhandlungen der Koniglich Pressischen Geologischen Landesanstalt, 9(2): 1-298.

Potonie, R. 1956. Synopsis der Gattungen der Sporae dispersae. I. Teil: Sporites. Beihefte zum Geologischen Jahrbuch, 23, 103 p.

Potonie, R. 1960. Synopsis der Gattungen der Sporae dispersat. III. Teil: Nachträge Sporites, Fortsetsung Pollenites, Mit Genereralregister zer Teil I-III. Geologisches Jahrbuch, Beihefte, 39: 1-189.

Premaor, E., Souza, P.A., Arai, M. \& Helenes, J. 2010. Palinomorfos do Campaniano (Cretáceo Superior) da Bacia de Pelotas, Rio Grande do Sul: implicações bioestratigráfica e paleoambientais. Pesquisas em Geociências, 37(1): 63-79.

Ramos, R.C. 2013. Caracterização Paleopalinológica da Formação Entre-Córregos, Bacia de Aiuruoca, MG, Brasil. Guarulhos, 70p. Trabalho de Conclusão de Curso, Curso deCiências Biológicas, Universidade Guarulhos.

Rao, M.R. 1995. Palynostratigraphy zonation and correlation of the Eocene-Early Miocene sequence in Alleppey district, Kerala, India. Review of Palaeobotany and Palynology, 86: 325-348.

Regali, M.S.P. 1971. Palinologia dos sedimentos cenozóicos da Foz do Rio Amazonas. São Paulo, 118p. Tese de Doutorado, Instituto de Geociências, Universidade de São Paulo.

Regali, M.S.P., Uesugui, N. \& Santos, A.S. 1974a. Palinologia dos Sedimentos Meso-Cenozóicos do Brasil (I). Boletim Técnico da Petrobrás, 17(3): 177-190.

Regali, M.S.P., Uesugui, N. \& Santos, A.S. 1974b. Palinologia dos Sedimentos Meso-Cenozóicos do Brasil (II). Boletim Técnico da Petrobrás, 17(4): 263-301.

Rull, V. 2001. A Quantitative Palynological Record from the Early Miocene of Western Venezuela, with Emphasis on Mangroves. Palynology, 25: 109-126.

Rull, V. 2003. Contribution of quantitative ecological methods to the interpretation of stratigraphically homogeneous Pre-Quaternary sediments: a palynological example from the Oligocene of Venezuela. Palynology, 27: 75-98.

Santos, D.B. 2005. Palinologia de amostras da seção-tipo da Formação Itaquaquecetuba, Bacia de São Paulo, na Mineradora Itaquareia I: implicações palinocrono-estratigráficas e paleoambientais. Guarulhos, 148p. Monografia de Conclusão de Curso, Curso de Ciências Biológicas, Universidade Guarulhos.

Santos, D.B., Garcia, M.J., Fernandes, R.S., Saad, A.R. \& Bistrichi, C.A. 2006a. Composição paleoflorística dos depósitos terciários da Formação Itaquaquecetuba, Estado de São Paulo, Brasil. In: SIMPÓSIO DO CRETÁCEO DO BRASIL, 7, SIMPÓSIO DO TERCIÁRIO DO BRASIL, 1, 2006, Serra Negra. Boletim de Resumos, UNESP, p. 20.

Santos, D.B., Garcia, M.J., Fernandes, R.S., Saad, A.R. \& 
Bistrichi, C.A. 2006b. A Paleopalinologia na reconstrução da paisagem terciária da Formação Itaquaquecetuba (Mineradora Itaquareia 1), Município de Itaquaquecetuba, Estado de São Paulo, Brasil. In: SIMPÓSIO ARGENTINO DE PALEOBOTANICA Y PALINOLOGÍA, 13, 2006, Bahia Blanca. Boletín de Resúmenes, Universidad Nacional del Sur y CONISET, p. 72.

Santos, D.B., Garcia, M.J., Saad, A.R. \& Bistrichi, C.A. 2010. Palinoestratigrafia da Formação Itaquaquecetuba, bacia de São Paulo, Brasil. Revista Brasileira de Paleontologia, 13(3): 205-220.

Scaphati, L., Melendi, D.L. \& Volkheimer. 2009. A Danian subtropical lacustrine palynobiota from South America (Bororó Formation, San Jorge Basin, Patagonia - Argentina). Geologica Acta, 7(1-2): 35-61.

Silva, W.G., Souza, P.A. \& Arai, M. 2011. Palinomorfos neógenos do poço 2-CA-1-RS, Bacia de Pelotas, Brasil: significado bioestratigráfico e paleoecológico. Geologia USP, Série Científica, 11: 149-169.

Silva-Caminha, S.A.F. da., Jaramillo, C.A. \& Absy, M.L. 2010. Neogene palynology of the Solimões Basin, Brazilian Amazônia. Palaeontographica Abteilung B, 283: 1-67.

Silveira, R.R. 2005. Cronoestratigrafia e interpretação paleoambiental de depósitos miocenos da Formação Solimões, região de Coari, AM. Manaus, 115p. Dissertação de Mestrado, Programa de Pós-Graduação em Geologia Regional, Instituto de Ciências Exatas, Universidade Federal do Amazonas.

Silveira, R.R. 2015 . Bioestratigrafia e paleoecologia de depósitos neógenos e pleistocenos aflorantes na porção central e ocidental da Bacia do Solimões, Estado do Amazonas, Brasil. Porto Alegre, 264p. Tese de Doutorado, Programa de Pós-Graduação em Geociências, Instituto de Geociências, Universidade Federal do Rio Grande do Sul.

Silveira, R.R. \& Nogueira, A.C.R. 2007. Cronoestratigrafia de Depósitos Miocenos da Bacia do Solimões e sua correlação com depósitos da Amazônia. In: CONGRESSO DA ABEQUA 2007, Belém. Boletim de Resumos, ABEQUA, Belém, v. 1, CDRom.

Silveira, R.R. \& Souza, P.A. 2015. Palinologia (grãos de pólen de angiospermas) das formações Solimões e Içá (Bacia do Solimões), nas regiões de Coari e Alto Solimões, Brasil. Revista Brasileira de Paleontologia, 18(3): 455-474.

Silveira, V.D. 1981. Micologia. 4a․ ed., Rio de Janeiro, Interamericana, 332p.

Singh, C. 1964. Microflora of the Lower Cretaceous Mannville Group, East-Central Alberta. Research Council of Alberta Bulletin, 15: 1-239.

Soares, E.A.A., Tatumi, S.H., Riccomini, C. 2010. OSL age determinations of Pleistocene fluvial deposits in Central Amazonia. Anais da Academia Brasileira de Ciências, 82: 1-9.

Tryon, R.M. \& Tryon, A.F. 1982. Ferns and Allied Plants. New York, Springer-Verlag, 648p.

Uesugui, N. 1979. Palinologia; técnicas de tratamento de amostras. Boletim Técnico da Petrobrás, 22: 229240.

van der Hammen, T. 1956. A palynological systematic nomenclature. Boletín Geológico, 4: 63-101.

van Geel, B. \& van der Hammen, T. 1978. Zygnemataceae in Quaternary Colombian sediments. Review of $\mathrm{Pa}$ laeobotany and Palynology, 25: 377-392.

Wanderley Filho, J.R., Eiras, J.F. \& Vaz, P.T. 2007. Bacia do Solimões. Boletim de Geociências Petrobrás, 15(2): 217-225.

Worobiec, E. 2009. Middle Miocene palynoflora of the Legnica lignite deposit complex, Lower Silesia, Poland. Acta Palaeobotanica, 49(1): 5-133.

Yamamoto, I.T. 1995. Palinologia das bacias tafrogênicas do Sudeste (bacias de Taubaté, São Paulo e Resende): análise bioestratigráfica integrada e interpretação paleoambiental. Rio Claro, 217p. Tese de Doutorado, Instituto de Geociências e Ciências Exatas, Universidade Estadual Paulista.

Man 557

Editores: Marina Bento Soares \& Maria do Carmo Lima e Cunha. 
Apêndice. Informações sobre a procedência dos espécimes de palinomorfos ilustrados (*codificações são referentes às coleções da Universidade Federal do Amazonas: Am, e Universidade Federal do Rio Grande do Sul: MP-P; ** coordenada England Finder).

\begin{tabular}{|c|c|c|c|c|}
\hline \multirow{2}{*}{$\begin{array}{l}\text { Táxon } \\
\text { Esporos de fungos }\end{array}$} & \multirow[t]{2}{*}{ Área e afloramento } & \multicolumn{2}{|c|}{ Lâmina* e coordenada EF** } & \multirow[t]{2}{*}{ Ilustração (Fig.) } \\
\hline & & & & \\
\hline Diporicellaesporites sp. 1 & Coari/P2 & Am13A & $\mathrm{Q} 50 / 1$ & 2.8 \\
\hline Diporicellaesporites sp. 2 & Coari/P2 & Am21A & $\mathrm{R} 57 / 4$ & 2.9 \\
\hline Esporo de fungo indeterminado & Coari/P2 & Am13A & $\mathrm{N} 60 / 1$ & 2.6 \\
\hline Foveodiporites sp. & Coari/P2 & Am13A & U56 & 2.4 \\
\hline Fusiformisporites crabbii & Alto Solimões/P5 & MP-P 10123 & Y48 & 2.5 \\
\hline Mediaverrunites elsekii & Coari/P2 & Am1A & $\mathrm{H} 26 / 1$ & 2.7 \\
\hline Meliolinites sp. & Coari/P2 & Am8A & $\mathrm{R} 35 / 2$ & 2.10 \\
\hline Monoporisporites sp. 1 & Coari/P2 & $\mathrm{Am} 12 \mathrm{~A}$ & $051 / 2$ & 2.1 \\
\hline Monoporisporites sp. 2 & Coari/P2 & Am5A & $\mathrm{L} 47 / 4$ & 2.2 \\
\hline Monoporisporites sp. 3 & Coari/P2 & Am13A & V03 & 2.3 \\
\hline Quilonia sp. & Coari/P12 & Am7A & D40/D41 & 2.11 \\
\hline \multicolumn{5}{|l|}{ Esporos de pteridófitas } \\
\hline Cicatricosisporites $\mathrm{sp}$. & Coari/P2 & Am9B & F29 & 4.8 \\
\hline Cingulatisporites sp. & Coari/P2 & Am9B & $\mathrm{D} 34 / 1$ & 3.8 \\
\hline Crassoretitriletes vanraadshoovenii & Alto Solimões/P1 & MP-P 6775 & $\mathrm{E} 47 / 3$ & $4.6 \mathrm{a}-\mathrm{b}$ \\
\hline Cyatheacidites annulatus & Coari/P2 & Am9B & $\mathrm{E} 35 / 1$ & 3.9 \\
\hline Psilatriletes peruanus & Coari/P2 & Am8A & $\mathrm{C} 32 / 4$ & 3.7 \\
\hline Cyathidites sp. 1 & Coari/P2 & Am1A & $\mathrm{F} 23 / 3$ & 3.3 \\
\hline Cyathidites sp. 2 & Coari/P2 & Am1B & W31/3 & 3.4 \\
\hline Cyathidites sp. 3 & Alto Solimões/P1 & MP-P 6765 & $\mathrm{E} 33 / 3$ & 3.2 \\
\hline Deltoidospora adriennis & Alto Solimões/P4 & MP-P 7518 & $\mathrm{R} 45$ & 3.1 \\
\hline Echitriletes muelleri & Coari/P2 & Am3A & Q37/2 & 4.9 \\
\hline Foveotriletes ornatus & Alto Solimões/P1 & MP-P 6767 & V48 & 3.6 \\
\hline Kuylisporites waterbolkii & Coari/P2 & Am1A & W35/3 & 3.5 \\
\hline Laevigatosporites sp. 1 & Coari/P13 & Am4B & $\mathrm{R} 17 / 1$ & 5.2 \\
\hline Laevigatosporites sp. 2 & Coari/P2 & Am10A & P39 & 5.3 \\
\hline Laevigatosporites sp. 3 & Coari/P2 & Am1A & $\mathrm{J} 25 / 1$ & 5.4 \\
\hline Laevigatosporites sp. 4 & Coari/P2 & Am1A & $\mathrm{K} 21$ & 5.5 \\
\hline Laevigatosporites vulgaris & Coari/P13 & Am8A & $\mathrm{B} 40 / 1$ & 5.1 \\
\hline Magnastriatites grandiosus & Alto Solimões/P1 & MP-P 6765 & $\mathrm{R} 27$ & 4.7 \\
\hline Perinomonoletes sp. & Coari/P2 & Am3A & $\mathrm{E} 32 / 1$ & 5.11 \\
\hline Polypodiaceiosporites potoniei & Coari/P2 & Am5B & R63 & $3.10 \mathrm{a}-\mathrm{b}$ \\
\hline P. pseudopsilatus & Coari/P2 & Am7A & $\mathrm{X} 24 / 4$ & $4.1 \mathrm{a}-\mathrm{b}$ \\
\hline Polypodiaceiosporites sp. & Coari/P2 & Am7A & $\mathrm{T} 21 / 4$ & 3.11 \\
\hline Polypodiisporites radiatus & Coari/P13 & Am8A & $\mathrm{Y} 40 / 3$ & 5.6 \\
\hline Striatriletes saccolomoides & Coari/P13 & $\mathrm{Am} 3 \mathrm{~A}$ & $\mathrm{~T} 20 / 3$ & $4.5 \mathrm{a}-\mathrm{b}$ \\
\hline Verrucatosporites favus & Coari/P2 & $\mathrm{Am} 2 \mathrm{~A}$ & W35/2 & 5.8 \\
\hline Verrucatosporites sp. 1 & Coari/P2 & Am8A & $\mathrm{D} 38 / 2$ & 5.9 \\
\hline Verrucatosporites sp. 2 & Coari/P2 & Am19A & S19 & 5.10 \\
\hline Verrucatosporites usmensis & Alto Solimões/P1 & MP-P 6765 & $\mathrm{~F} 52 / 1$ & 5.7 \\
\hline Verrucatotriletes bullatus & Coari/P2 & Am5B & R52 & 4.4 \\
\hline Verrucatotriletes sp.1 & Coari/P2 & Am $1 \mathrm{~A}$ & K22 & 4.2 \\
\hline Verrucatotriletes sp. 2 & Coari/P2 & Am5B & $\mathrm{R} 65 / 3$ & 4.3 \\
\hline \multicolumn{5}{|l|}{ Grão de pólen de gimnospermas } \\
\hline Podocarpidites sp. 1 & Alto Solimões/P7 & MP-P 7740 & L49/3 & 5.8 \\
\hline Podocarpidites sp. 2 & Coari/P2 & $\mathrm{Am} 3 \mathrm{~A}$ & $\mathrm{E} 26 / 4$ & 5.9 \\
\hline \multicolumn{5}{|l|}{ Algas clorofíceas } \\
\hline Ovoidites sp. & Coari/P2 & Am7A & $\mathrm{V} 23 / 3$ & 6.7 \\
\hline Pediastrum sp. 1 & Alto Solimões/P7 & MP-P 7730 & S46 & 6.1 \\
\hline Pediastrum sp. 2 & Alto Solimões/P7 & MP-P 7730 & $\mathrm{P} 53 / 3$ & 6.2 \\
\hline Pediastrum sp. 3 & Alto Solimões/P7 & MP-P 7730 & F49 & 6.3 \\
\hline Pediastrum sp. 4 & Alto Solimões/P7 & MP-P 7730 & E28 & 6.4 \\
\hline Pseudoschizaea circulus & Coari/P2 & Am5A & $\mathrm{T} 36 / 4$ & 6.5 \\
\hline Pseudoschizaea sp. & Coari/P2 & Am13A & $\mathrm{H} 42 / 1$ & 6.6 \\
\hline \multicolumn{5}{|l|}{ Alga dinofícea } \\
\hline Diphyes sp. & Alto Solimões/P7 & MP-P 7740 & L49/3 & 6.9 \\
\hline \multicolumn{5}{|l|}{ Fragmento de invertebrado } \\
\hline Escolecodonte indeterminado & Coari/P2 & $\mathrm{Am} 3 \mathrm{~A}$ & E26/4 & 6.8 \\
\hline
\end{tabular}


